\title{
Study of exotic hadrons in $S$-wave scatterings induced by chiral interaction in the flavor symmetric limit
}

\author{
Tetsuo Hyodd* and Daisuke Jido \\ Yukawa Institute for Theoretical Physics, Kyoto University, Kyoto 606-8502, Japan \\ Atsushi Hosaka \\ Research Center for Nuclear Physics (RCNP), Ibaraki, Osaka 567-0047, Japan
}

(Dated: July 10, 2018)

\begin{abstract}
We study $s$-wave bound states of a hadron and a light pseudoscalar meson induced by the Weinberg-Tomozawa (WT) interaction in the flavor SU(3) symmetric limit. The WT interaction is a driving force to generate quasibound states dynamically in the chiral unitary approaches. The strength and sign of the WT interaction are determined only by the group theoretical structure of the target hadrons, and we present a general expression of the strengths for the flavor SU(3) case. We show that, for the channels which are more exotic than the target, the interaction is repulsive in most cases, and the strength of the attractive interaction is universal for any possible target states. We demonstrate that the attractive coupling is not strong enough to generate an exotic state from the physically known masses of target hadrons. In addition, we also find a nontrivial $N_{c}$ dependence of the coupling strengths. We show that the channels which are attractive at $N_{c}=3$ changes into repulsive ones for large $N_{c}$, and, therefore, no attractive interaction exists in exotic channels in the large- $N_{c}$ limit.

PACS numbers: 14.20.-c, 11.30.Rd, 11.30.Hv, 11.15.Pg
\end{abstract}

\section{INTRODUCTION}

The hadrons observed in experiments are classified by their spin and flavor quantum numbers [1]. The flavor quantum numbers are ordinarily composed of the valence quark contents of $\bar{q} q$ for mesons and $q q q$ for baryons. The mesons and baryons with flavor that cannot be achieved by $\bar{q} q$ or $q q q$ are called exotic hadrons, and must have more valence quarks than the ordinary hadrons. So far, the existence of the exotic state has not been clearly established. The evidence for the pentaquark $\Theta^{+}$at LEPS/SPring8 [2] is one of the possible candidates, but the experimental confirmation of the existence of $\Theta^{+}$is still controversial [3]. The structure of the $X(3872)$ [4] and $D_{s}(2317)[5]$ is also found to be quite different from a simple $\bar{q} q$ state, although their flavor quantum numbers can be achieved by $\bar{q} q$.

The definitive answer for whether the exotics exist or not will be given by future experiments, but it is highly nontrivial that such states are almost completely absent in the hadron spectrum. The fundamental theory of the strong interaction, QCD, does not forbid the exotic states. There is no simple selection rule to exclude such states in effective models describing the ordinary hadrons well. In this context, we have studied the nature of the exotic states as hadronic molecule states in Ref. [6] based on the $s$-wave dynamics with the underlying chiral symmetry in QCD.

In recent years, there have been remarkable developments in theoretical studies of hadron spectroscopy.

\footnotetext{
*Electronic address: hyodo@yukawa.kyoto-u.ac.jp
}

Some of the baryon resonances are well described as meson-baryon quasibound states in chiral unitary approaches [7, 8, 9, 10, 11]. These resonances are dynamically generated in meson-baryon scattering formulated by unitarization of low energy interactions governed by chiral symmetry. This approach is along the same lines as the coupled-channel analysis developed in the 1960's [12, 13], and recent studies revealed the novel structure of the $\Lambda(1405)$, namely, the existence of two poles in the region of $\Lambda(1405)[14,15,16]$. The method was also applied to the scattering of the pseudoscalar mesons with the decuplet baryons of $J^{P}=3 / 2^{+}$, leading to the dynamical generation of the $J^{P}=3 / 2^{-}$resonances [17, 18]. Applications to the charmed baryon sector [19] and the heavy meson sector [20, 21, 22] were also performed, in fair agreement with the experimental observation. In these studies, an important ingredient to generate $s$-wave resonances dynamically was the Weinberg-Tomozawa (WT) interaction of hadrons and the Nambu-Goldstone (NG) bosons [23, 24].

The WT term has unique features in chiral dynamics. The kinematical structure of the coupling is fixed as an $s$-wave interaction between the target hadron and NG boson system with linear dependence of the NG boson energy. Also, its sign and strength are universally determined by chiral symmetry, once one fixes the flavor structure of the hadron. This is because the interaction can be derived from the current algebra, even without using the chiral Lagrangians. In addition, the WT term is the leading order term of the chiral expansion, and therefore it gives the dominant contribution at low energy. Thus, the low energy hadron interaction in an $s$-wave is governed by the WT term, which is model independent, as far as we respect chiral symmetry. 
In this paper, we would like to present a detailed analysis of Ref. [6], studying the mechanism of the generation of resonances of the NG boson and a target hadron in a simplified and essential version of the chiral unitary approaches, paying attention to the flavor exotic states. The dynamically generated state in this approach is considered as a quasibound state of the NG boson and the target hadron. Since such a generated state has additional $\bar{q} q$ components, it is natural to consider exotic states in this approach. To start with, we take flavor $\mathrm{SU}(3)$ symmetric limit for simplicity. In the $\mathrm{SU}(3)$ limit, the complicated coupled-channel equations reduce to a set of independent single-channel problems. In Refs. 14, 25], some resonances in the physical world of $\mathrm{SU}(3)$ breaking were shown to turn into bound states in the limit of SU(3) symmetry. The existence of the bound states in the $\mathrm{SU}(3)$ limit was also confirmed in different channels [17, 18, 19, 20]. Therefore, in this limit, our task is to search for bound state poles in the scattering amplitude on the real axis below threshold, which are considered to be the origin of the physical resonances with $\mathrm{SU}(3)$ breaking.

This paper is organized as follows. In Sec. II, we review the formulation of the chiral unitary approach and show how the physical resonances are dynamically generated. In Sec. III. we focus on the WT term, that is, the kernel interaction of the chiral unitary approach. Based on a group theoretical argument, we present the general formula of the coupling strength of the WT term. Its expressions give us the important consequence that the interaction in exotic channels is repulsive in most cases, and the possible strength of the attraction is independent of channels. The behavior of the coupling strength in the large- $N_{c}$ limit is also discussed. In Sec. IV] we derive the condition to generate a bound state, based on the general principle of the scattering theory. Presenting numerical analysis with physical masses and coupling constants, we show under which condition the bound states are generated in the exotic channels. The last section is devoted to a summary.

\section{CHIRAL UNITARY APPROACH}

In this section, we present the formulation of the chiral unitary approach that describes the scattering of a NG boson with a target hadron. There are two important ingredients, chiral symmetry of the interaction and the unitarity of the scattering amplitude. The scattering amplitude is nonperturbatively constructed so as to maintain the unitarity and to match the kernel interaction with the chiral perturbation theory in the low energy limit. The resonances can be dynamically generated in the resulting amplitude which agrees well with experimental data for various target hadrons.

\section{A. Kernel interaction}

Here we introduce the Weinberg-Tomozawa interaction [23, 24], focusing on the kinematic structure. To start with, let us take an example of the scattering of the pseudoscalar octet mesons (the NG bosons of three flavor chiral symmetry) with the ground state octet baryons. In the chiral perturbation theory, the WT interaction is derived as the leading order term of the chiral expansion in the covariant derivative of the kinetic term,

$\operatorname{Tr}(\bar{B} i \not D B)=\operatorname{Tr}(\bar{B} i \not \partial B)+\operatorname{Tr}\left(\bar{B} i \gamma^{\mu} \frac{1}{4 f^{2}}\left[\Phi \overleftrightarrow{\partial_{\mu}} \Phi, B\right]\right)+\ldots$

with the meson decay constant $f$ in the $\mathrm{SU}(3)$ limit and the baryon octet $(B)$ and the pseudoscalar meson octet $(\Phi)$ fields [26, 27, 28, 29]. This term provides the interaction of the meson-baryon scattering

$$
V_{i j}^{(W T)}=-\frac{1}{4 f^{2}} C_{i j} \bar{u}\left(\not k_{i}+\not k_{j}\right) u,
$$

where $i, j$ denote the meson-baryon channels in the final and initial states, $k_{i}$ and $k_{j}$ are the momenta of the meson in channels $i$ and $j$, respectively, $u$ and $\bar{u}$ are the baryon spinors, and $C_{i j}$ is the coupling strength matrix in channel space.

The sign and strength of the matrix element $C_{i j}$ are determined by the flavor symmetry. In general, the matrix $C_{i j}$ has the off-diagonal components which are responsible for the transition between two different channels. Therefore, solving the scattering equation is a coupledchannel problem. On the other hand, $C_{i j}$ becomes a diagonal matrix in the basis of the $\mathrm{SU}(3)$ irreducible representations, since the interaction is $\mathrm{SU}(3)$ symmetric. Using the $\mathrm{SU}(3)$ Clebsch-Gordan coefficients $\langle i, \alpha\rangle$ which relates the particle basis $i$ with the $\mathrm{SU}(3)$ basis $\alpha$ symbolically, the matrix $C_{i j}$ can be transformed into that in the $\mathrm{SU}(3)$ basis as $C_{\alpha \beta}=\sum_{i, j}\langle i, \alpha\rangle C_{i j}\langle j, \beta\rangle=C_{\alpha} \delta_{\alpha \beta}$, where $\alpha$ and $\beta$ denote the irreducible representations of $\mathrm{SU}(3)$ [14]. Since this matrix is diagonal in the $\mathrm{SU}(3)$ basis, the coupled-channel equations reduce to a set of independent single-channel equations.

In the $\mathrm{SU}(3)$ limit, the interaction is written in the nonrelativistic reduction as

$$
V_{\alpha \beta}^{(W T)} \sim-\frac{\omega}{2 f^{2}} C_{\alpha, T} \delta_{\alpha \beta},
$$

with the energy of the meson $\omega$ and the diagonal matrix $C_{\alpha, T}$ for which we put the index of the target representation $T$ for later convenience.

It is important to note that the structure and strength of the WT term are determined by chiral symmetry without introducing additional coupling constants; the constant $f$ can be determined by the weak decay of the NG boson. In addition, the structure of the coupling is universally fixed for any target states, since Eq. (2) is also derived from current algebra, without using chiral Lagrangian [6]. Therefore, although Eq. (10) is given for the 
system of the NG boson and the octet baryons, we can generalize Eq. (2) to that of any hadron targets. For a meson target, some minor modifications should be made in Eq. (2) and the formulas of this section. Nevertheless, we find that the expression given in this section can be applied to the meson target in the heavy mass approximation. The detailed discussion is given in Appendix C

\section{B. Unitarization and regularization}

With the WT interaction in the perturbation theory, the scattering amplitude does not satisfy the unitarity condition. Here we show the way to maintain the unitarity [10, 30]. It necessarily brings a regularization procedure for divergent loop integrals [11]. We again take the example of the meson-baryon scattering.

In the chiral unitary approaches, we impose the unitarity condition for the scattering amplitude, based on the N/D method [29]. Assuming the elastic unitarity and neglecting the left-hand cut from the crossed diagrams, the meson-baryon scattering amplitude reads

$$
t(\sqrt{s})=\frac{1}{1-V(\sqrt{s}) G(\sqrt{s})} V(\sqrt{s}),
$$

as a function of the center-of-mass energy $\sqrt{s}$. Here $V(\sqrt{s})$ denotes the WT interaction (2) where $\omega=(s-$ $\left.M_{T}^{2}+m^{2}\right) /(2 \sqrt{s})$ with $m$ and $M_{T}$ being masses of the meson and baryon, respectively. The function $G(\sqrt{s})$ is given by the spectral representation

$$
G(\sqrt{s})=-\tilde{a}\left(s_{0}\right)-\frac{1}{2 \pi} \int_{s^{+}}^{\infty} d s^{\prime}\left(\frac{\rho\left(s^{\prime}\right)}{s^{\prime}-s}-\frac{\rho\left(s^{\prime}\right)}{s^{\prime}-s_{0}}\right),
$$

where $s^{+}=\left(m+M_{T}\right)^{2}$ is the value of $s$ at the threshold. The parameter $\tilde{a}\left(s_{0}\right)$ is the subtraction constant which is not determined within the N/D method. The integrated phase space is

$$
\rho(s)=\frac{2 M_{T} \bar{q}}{4 \pi \sqrt{s}},
$$

where $\bar{q}=\lambda^{1 / 2}\left(s, M_{T}^{2}, m^{2}\right) / 2 \sqrt{s}$ and the Källen function $\lambda^{1 / 2}(x, y, z)=x^{2}+y^{2}+z^{2}-2 x y-2 y z-2 z x$.

In principle, the renormalization of the amplitude should be performed so as to reproduce some experimental observables. Here, to fix the subtraction parameter, we adopt the prescription given in Ref. [11], which gives the proper subtraction constant for the $S=-1$ mesonbaryon channel as we will see below. In this scheme, the scattering amplitude is adjusted to be the kernel interaction $V$ at $\sqrt{s}=M_{T}$ with the regularization scale $\mu=M_{T}$ :

$$
G(\mu)=0 \quad \Leftrightarrow \quad t(\mu)=V(\mu) \quad \text { at } \mu=M_{T} .
$$

This is equivalent to taking the subtraction point at the baryon mass:

$$
s_{0}=M_{T}^{2}, \quad \tilde{a}\left(M_{T}^{2}\right)=0 .
$$

This condition determines the energy at which the chiral perturbation theory works. At the same time, this condition guarantees that the present amplitude with $s$ channel unitarity coincides with the crossed amplitude with $u$-channel unitarity at $\sqrt{s}=M_{T}$. This prescription was first introduced in the $\pi-\pi$ scattering [31], and was applied to the meson-baryon scattering in Ref. [11].

It is instructive to show that the algebraic form of Eq. (3) derived in the N/D method was proved to be equivalent to the solution of the on-shell factorized BetheSalpeter integral equation [10] if one identifies the $G$ function (4) with the meson-baryon loop function,

$$
\begin{aligned}
G(\sqrt{s})= & i \int \frac{d^{4} q}{(2 \pi)^{4}} \frac{2 M_{T}}{(P-q)^{2}-M_{T}^{2}+i \epsilon} \frac{1}{q^{2}-m^{2}+i \epsilon} \\
= & \frac{2 M_{T}}{(4 \pi)^{2}}\left\{a(\mu)+\ln \frac{M_{T}^{2}}{\mu^{2}}+\frac{m^{2}-M_{T}^{2}+s}{2 s} \ln \frac{m^{2}}{M_{T}^{2}}\right. \\
& +\frac{\bar{q}}{\sqrt{s}}\left[\ln \left(s-\left(M_{T}^{2}-m^{2}\right)+2 \sqrt{s} \bar{q}\right)\right. \\
& +\ln \left(s+\left(M_{T}^{2}-m^{2}\right)+2 \sqrt{s} \bar{q}\right) \\
& -\ln \left(-s+\left(M_{T}^{2}-m^{2}\right)+2 \sqrt{s} \bar{q}\right) \\
& \left.\left.-\ln \left(-s-\left(M_{T}^{2}-m^{2}\right)+2 \sqrt{s} \bar{q}\right)\right]\right\},
\end{aligned}
$$

where $P^{\mu}=(\sqrt{s}, \mathbf{0}), \mu$ and $a(\mu)$ are the regularization scale and subtraction constant, which correspond to $s_{0}$ and $\tilde{a}\left(s_{0}\right)$ in Eq. (4). This loop function is exactly the same as the dispersion integral (44) with the phase space (5) up to constants.

The renormalization condition (6) is suitable for the present purpose, since the regularization parameters $a$ and $\mu$ are systematically fixed by the baryon and meson masses without other experimental inputs:

$$
\begin{aligned}
\mu= & M_{T}, \\
a\left(M_{T}\right)= & -\left\{\frac{m^{2}}{2 M_{T}^{2}} \ln \frac{m^{2}}{M_{T}^{2}}+\frac{m \sqrt{m^{2}-4 M_{T}^{2}}}{2 M_{T}^{2}}\right. \\
& \times\left[\ln \left(m^{2}+m \sqrt{m^{2}-4 M_{T}^{2}}\right)\right. \\
& +\ln \left(2 M_{T}^{2}-m^{2}+m \sqrt{m^{2}-4 M_{T}^{2}}\right) \\
& -\ln \left(-m^{2}+m \sqrt{m^{2}-4 M_{T}^{2}}\right) \\
& \left.\left.-\ln \left(-2 M_{T}^{2}+m^{2}+m \sqrt{m^{2}-4 M_{T}^{2}}\right)\right]\right\} .
\end{aligned}
$$

This is not the unique choice of the subtraction parameter. Generally, the subtraction constant is fixed at the renormalization point by experimental inputs, such as the scattering length. One can also determine $a(\mu)$ so as to reproduce the observed threshold branching ratios in the strangeness $S=-1$ channels [8, 10, 32], where $a(630 \mathrm{MeV}) \simeq-2$ is obtained. This is quite consistent with the subtraction constant obtained by our procedure with the averaged mass of the ground state octet baryons $M_{T}=1151 \mathrm{MeV}$ :

$$
a(630 \mathrm{MeV}) \sim-1.98,
$$


where we have used $a\left(\mu^{\prime}\right)=a(\mu)+2 \ln \left(\mu^{\prime} / \mu\right)$ 29]. In this way, we consider that the present renormalization scheme provides a natural extension to the channels in which the scattering observables are not available.

\section{Dynamically generated states and SU(3) limit}

When a baryon resonance $R$ is dynamically generated, the scattering amplitude has a pole in the second Riemann sheet of the complex energy plane. We can extract information of the resonance from the pole, by identifying the scattering amplitude close to the resonance energy region by the Breit-Wigner amplitude plus nonresonant background term:

$$
-i t_{i j}(\sqrt{s})=-i g_{i} \frac{i}{\sqrt{s}-M_{R}+i \Gamma_{R} / 2}\left(-i g_{j}\right)-i t_{i j}^{B G},
$$

for an $s$-wave resonance in the coupled-channel scattering with mass $M_{R}$ and total width $\Gamma_{R}$, where $g_{i}$ is the coupling strength of the resonance $R$ to the channel $i$. Since the NG boson is scattered in an $s$-wave, dynamically generated states have the same spin but opposite parity with the target hadron.

In the literature, many experimentally observed resonances were identified as hadronic molecule states generated dynamically in the scattering of the NG boson and hadrons. The studies with the octet baryon target successfully reproduced the $J^{P}=1 / 2^{-}$resonances $\Lambda(1405)$, $\Lambda(1670), \Sigma(1620)$ [32], $N(1535)$ [33], and $\Xi(1620)$ 34]. In a different scheme [25], the $\Xi(1690)$ resonance was found in addition to the $\Xi(1620)$. In the scattering with baryon decuplet target [17, 18], the $J^{P}=3 / 2^{-}$resonances such as $\Lambda(1520), \Xi(1820), \Sigma(1670)$ were well reproduced. In the heavy sector, charmed resonances $\Lambda_{c}(2880)$ and $\Lambda_{c}(2593)$ were generated dynamically in the scattering of the NG boson with the ground state charmed baryons [19]. For the heavy meson sector, the recently discovered $D_{s}(2317)$ was properly reproduced as a resonance of the NG boson and the ground state charmed mesons [20].

It was shown that the resonances found in the light baryon sectors became bound states in the SU(3) limit, by tracing the positions of poles with gradual restoration of SU(3) symmetry 14, 18, 25]. The existence of the bound state poles is also observed in the heavy sectors [19, 20]. These facts imply that the bound states are first generated in the $\mathrm{SU}(3)$ limit; then they acquire widths through the coupled-channel dynamics in the different thresholds among the channels due to the $\mathrm{SU}(3)$ breaking. Therefore, in order to clarify the mechanism to generate physical resonances, we study the bound states in the $\mathrm{SU}(3)$ limit, which are expected to be the origin of the resonances observed in nature.

In the next section, based on the group theoretical argument, we first study the sign and coupling strength of the WT interaction systematically for various channels, which is the driving force to generate the bound state. Of particular interest are the flavor exotic channels, where a small attraction will be found. Then we discuss whether the bound states can be generated with the given target hadron mass and the coupling strength obtained in the group theoretical argument.

\section{WEINBERG-TOMOZAWA INTERACTION}

\section{A. Group theoretical structure}

Here we discuss the interaction strengths $C_{\alpha, T}$ in Eq. (2). $C_{\alpha, T}$ is the interaction strength of the WT term in the channel belonging to the $\mathrm{SU}(3)$ irreducible representation $\alpha$ in the direct product space of the target hadron $T$ and the NG boson Ad as shown in Fig. 1(a). The interaction strength is dependent only on the representations of the channel $\alpha$, the hadron $T$ and the NG boson Ad which is always fixed as the adjoint representation. The WT term is the vector-current-vector-current interaction, so that $C_{\alpha, T}$ (with proper normalization) can be written as

$$
C_{\alpha, T}=-2\left\langle[\mathrm{Ad}, T]_{\alpha}\left|\boldsymbol{F}_{T} \cdot \boldsymbol{F}_{\mathrm{Ad}}\right|[\mathrm{Ad}, T]_{\alpha}\right\rangle,
$$

where $\boldsymbol{F}_{T}$ and $\boldsymbol{F}_{\mathrm{Ad}}$ are the SU(3) generators in the representations of the hadron $T$ and the NG boson Ad, respectively. The state labelled by $[\mathrm{Ad}, T]_{\alpha}$ belongs to the representation $\alpha$ composed of the two particle system Ad $\otimes T$, which is schematically shown in Fig. 1(a). The bound states appearing in the channel $\alpha$ after the unitarization of the amplitude has the flavor quantum number $\alpha$, as depicted in Fig. 1(b).

The matrix element of Eq. (8) can be expressed in terms of Casimir invariants:

$$
\begin{aligned}
& \left\langle[\mathrm{Ad}, T]_{\alpha}\left|\boldsymbol{F}_{T} \cdot \boldsymbol{F}_{\mathrm{Ad}}\right|[\mathrm{Ad}, T]_{\alpha}\right\rangle \\
& \quad=\frac{1}{2}\left[C_{2}(\alpha)-C_{2}(\mathrm{Ad})-C_{2}(T)\right],
\end{aligned}
$$

where $C_{2}(R)$ is the quadratic Casimir of the representation $R$. This is consistent with the fact that the WT term is invariant under the $\mathrm{SU}(3)$ transformation. Recalling $C_{2}(\mathrm{Ad})=3$ for the adjoint representation, we find that the strength of the WT interaction can be written as

$$
C_{\alpha, T}=C_{2}(T)-C_{2}(\alpha)+3 .
$$

(a)

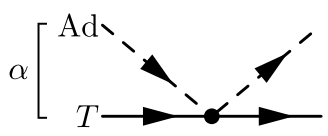

(b)

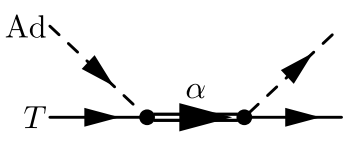

FIG. 1: (a): Notation of the representations $\alpha, \mathrm{Ad}$, and $T$ for the WT term. (b): The bound state pole diagram after unitarization of the amplitude. 
As seen in Eq. (2), the potential is proportional to $-C_{\alpha, T}$, and therefore a negative $C_{\alpha, T}$ leads to a repulsive interaction, whereas a positive $C_{\alpha, T}$ gives an attractive interaction in the present convention. The quadratic Casimir for the $[p, q]$ representation in the tensor notation is given by

$$
C_{2}([p, q])=\frac{1}{3}\left[p^{2}+q^{2}+p q+3(p+q)\right] .
$$

In the $\mathrm{SU}(2)$ case, Eq. (9) is expressed as

$$
C_{\alpha, T}^{\mathrm{SU}(2)}=-\left[I_{\alpha}\left(I_{\alpha}+1\right)-I_{T}\left(I_{T}+1\right)-2\right],
$$

where $I_{\alpha}$ is the total isospin of the $\pi$-T state, and $I_{T}$ is the isospin of the target. This is well-known expression of the $\pi N$ scattering lengths of the Weinberg-Tomozawa relation [23, 24].

It is worth noting that, for the $\mathrm{SU}(3)$ case, the $C_{i j}$ in the isospin space are given by Eq. (9) and the $\mathrm{SU}(3)$ isoscalar factors [35, 36] with suitable phase conventions:

$$
\begin{aligned}
& C_{i j}\left(I, I_{M_{i}}, Y_{M_{i}}, I_{T_{i}}, Y_{T_{i}}, I_{M_{j}}, Y_{M_{j}}, I_{T_{j}}, Y_{T_{j}}\right) \\
& =\sum_{\alpha}\left[C_{2}(T)-C_{2}(\alpha)+3\right]\left(\begin{array}{cc}
8 & T \\
I_{M_{i}}, Y_{M_{i}} & I_{T_{i}}, Y_{T_{i}}
\end{array} \| \begin{array}{c}
\alpha, Y
\end{array}\right) \\
& \quad \times\left(\begin{array}{cc}
8 & T \\
I_{M_{j}}, Y_{M_{j}} & I_{T_{j}}, Y_{T_{j}}
\end{array} \| \begin{array}{c}
\alpha \\
I, Y
\end{array}\right), \\
& Y=Y_{M_{i}}+Y_{T_{i}}=Y_{M_{j}}+Y_{T_{j}},
\end{aligned}
$$

where $I_{X_{i}}$ and $Y_{X_{i}}$ are the isospin and hypercharge of the state $X$ in channel $i$.

\section{B. General expression for coupling strength and exoticness}

To form a bound state of a two-body system, the coupling should at least be attractive, $C_{\alpha, T}>0$, namely $C_{2}(\alpha)<3+C_{2}(T)$. This indicates the difficulty of generating exotic resonances from a nonexotic target, since the exotic states are generally in higher dimensional representations, and their Casimir invariants have larger values than those of the simple nonexotic hadrons.

Here we evaluate the coupling strengths $C_{\alpha, T}$, using Eqs. (9) and (10). We first consider the target hadrons in specific representations, such as octet and decuplet. We then consider the general cases with arbitrary representations of the target hadrons.

Let us consider the light flavor baryons, where the lowest lying hadrons are in octet $(N, \Lambda, \Sigma, \Xi)$ and decuplet $\left(\Delta, \Sigma^{*}, \Xi^{*}, \Omega\right)$ representations. The representations for $\alpha$ are found in the irreducible decomposition $T \otimes \mathbf{8}=\sum \alpha$. The coupling strengths for the octet $T=\mathbf{8}$ and decuplet $T=\mathbf{1 0}$ targets are obtained by (9) and (10) and summarized in Table [1] they were also reported in Refs. [14, 17, 18]. For light flavor baryons, nonexotic representations are the singlet, octet and decuplet representations, since they can be achieved by the three light quarks qqq: $\mathbf{3} \otimes \mathbf{3} \otimes \mathbf{3}=\mathbf{1} \oplus \mathbf{8} \oplus \mathbf{8} \oplus \mathbf{1 0}$. Thus, for the
TABLE I: The coupling strengths $C_{\alpha, T}$ for light flavor baryons.

\begin{tabular}{ccccccc}
\hline \hline$\alpha$ & $\mathbf{1}$ & $\mathbf{8}$ & $\mathbf{1 0}$ & $\overline{\mathbf{1 0}}$ & $\mathbf{2 7}$ & $\mathbf{3 5}$ \\
\hline$T=\mathbf{8}$ & 6 & 3 & 0 & 0 & -2 & \\
$T=\mathbf{1 0}$ & & 6 & 3 & & 1 & -3 \\
\hline \hline
\end{tabular}

light flavor baryons, $\alpha=\overline{\mathbf{1 0}}, \mathbf{2 7}$, and $\mathbf{3 5}$ are the exotic channels. We find that, among exotic channels, only the channel $\mathbf{2 7}$ composed by the decuplet target is attractive, $C_{\mathbf{2 7}, \mathbf{1 0}}=1>0$ [37], while the couplings in other exotic channels are either repulsive or zero.

We can apply the same argument to hadrons with heavy quarks. In the $\mathrm{SU}(3)$ group argument, the heavy quarks are spectators. For the hadrons containing one heavy quark $Q$, the nonexotic baryons $q q Q$ are classified in $\overline{\mathbf{3}}$ and $\mathbf{6}$, and the mesons $q \bar{Q}$ are in $\mathbf{3}$. The representation $\mathbf{3}$ is also used for baryons with two heavy quarks, which are not experimentally well established to construct a multiplet. The established lowest lying hadrons are classified in these nonexotic multiplets: charmed baryons $\overline{\mathbf{3}}\left(\Lambda_{c}, \Xi_{c}\right)$ and $\mathbf{6}\left(\Sigma_{c}, \Xi_{c}^{*}, \Omega_{c}\right)$, charmed mesons $\mathbf{3}\left(D, D_{s}\right)$, and bottomed mesons $\mathbf{3}\left(B, B_{s}\right)$. With these states being target hadrons, coupling strengths $C_{\alpha, T}$ are evaluated as shown in Table [1] For heavy baryons, $\overline{\mathbf{1 5}}$ and $\mathbf{2 4}$ are the exotic channels, while $\overline{\mathbf{6}}$ and $\mathbf{1 5}$ are the exotic states for the heavy mesons. We find that, as seen in Table III the interaction in the exotic channel 15 composed of the $\mathbf{6}$ heavy baryon is attractive, $C_{\overline{\mathbf{1 5}, 6}}=1>0$ [19], and the mesonic channel 6 from the 3 heavy meson target is also attractive, $C_{\overline{\mathbf{6}}, \mathbf{3}}=1>0[20]$. Note that the states in $\overline{\mathbf{1 5}}$ and $\mathbf{2 4}$ are flavor exotic with one heavy quark, which are different from the $\Theta_{c}$ state of charm -1 discussed in Refs. 38, 39, 40], since it has one anticharm quark.

It is interesting that the attractive interactions for the exotic channels are found only in the limited cases $(\alpha, T)=(\mathbf{2 7}, \mathbf{1 0}),(\overline{\mathbf{1 5}}, \mathbf{6})$, and $(\overline{\mathbf{6}}, \mathbf{3})$ with a universal strength $C_{\alpha, T}=1$. In the following, we show that this observation is true for the general targets with arbitrary representations.

Let us consider an arbitrary representation $T=[p, q]$ for the target hadron. Possible representations $\alpha$ for the hadron and NG boson system are obtained in the irreducible decomposition of the direct product of the $[p, q]$ for the target hadron and the adjoint $[1,1]$ for the NG

TABLE II: The coupling strengths $C_{\alpha, T}$ for heavy flavor hadrons.

\begin{tabular}{cccccccc}
\hline \hline$\alpha$ & $\overline{\mathbf{3}}$ & $\mathbf{6}$ & $\overline{\mathbf{1 5}}$ & $\mathbf{2 4}$ & $\mathbf{3}$ & $\overline{\mathbf{6}}$ & $\mathbf{1 5}$ \\
\hline$T=\overline{\mathbf{3}}$ & 3 & 1 & -1 & & & & \\
$T=\mathbf{6}$ & 5 & 3 & 1 & -2 & & & \\
$T=\mathbf{3}$ & & & & & 3 & 1 & -1 \\
\hline \hline
\end{tabular}


TABLE III: Properties of the WT interaction for various channels of representations $\alpha$ formed by a target hadron $[p, q]$ and an octet meson $[1,1]$. Tabulated from the left to right column are the channel representation $\alpha$, the condition to have $\alpha$ in the irreducible decomposition, the coupling strengths of the WT term $C_{\alpha, T}$ and its sign, the differences of the exoticness $E$, $\epsilon$, $\nu$ between $\alpha$ and $T$, the coupling strengths with arbitrary $N_{c}, C_{\alpha, T}\left(N_{c}\right)$, and the sign of the WT interaction at the large- $N_{c}$ limit.

\begin{tabular}{|c|c|c|c|c|c|c|c|c|}
\hline$\alpha$ & Condition & $C_{\alpha, T}$ & Sign & $\Delta E$ & $\Delta \epsilon$ & $\Delta \nu$ & $C_{\alpha, T}\left(N_{c}\right)$ & $V\left(N_{c} \rightarrow \infty\right)$ \\
\hline$[p+1, q+1]$ & - & $-p-q$ & Repulsive & 1 or 0 & 1 & 0 & $\frac{3-N_{c}}{2}-p-q$ & Repulsive \\
\hline$[p+2, q-1]$ & $q \geq 1$ & $1-p$ & $\ldots$ & 1 or 0 & 0 & 1 & $1-p$ & 0 \\
\hline$[p-1, q+2]$ & $p \geq 1$ & $1-q$ & $\ldots$ & 1 or 0 & 1 & -1 & $\frac{5-N_{c}}{2}-q$ & Repulsive \\
\hline$[p, q]$ & $q \geq 1$ & 3 & Attractive & 0 & 0 & 0 & 3 & 0 \\
\hline$[p, q]$ & $p \geq 1$ & 3 & Attractive & 0 & 0 & 0 & 3 & 0 \\
\hline$[p+1, q-2]$ & $q \geq 2$ & $3+q$ & Attractive & 0 or -1 & -1 & 1 & $\frac{3+N_{c}}{2}+q$ & Attractive \\
\hline$[p-2, q+1]$ & $p \geq 2$ & $3+p$ & Attractive & 0 or -1 & 0 & -1 & $3+p$ & 0 \\
\hline$[p-1, q-1]$ & $p \geq 1, q \geq 1$ & $4+p+q$ & Attractive & 0 or -1 & -1 & 0 & $\frac{5+N_{c}}{2}+p+q$ & Attractive \\
\hline
\end{tabular}

boson:

$$
\begin{aligned}
{[p, q] \otimes[1,1]=} & {[p+1, q+1] \oplus[p+2, q-1] } \\
& \oplus[p-1, q+2] \oplus[p, q] \oplus[p, q] \\
& \oplus[p+1, q-2] \oplus[p-2, q+1] \\
& \oplus[p-1, q-1] .
\end{aligned}
$$

There are maximally eight representations. It is understood that the representation $[a, b]$ with $a<0$ or $b<0$ is not taken into account, and that, for two $[p, q]$ representations, one has the condition $p \geq 1$ and the other has $q \geq 1$. The coupling strengths $C_{\alpha, T}$ are summarized in the third column of Table III. This is the general expression for the coupling strength of the WT interaction for arbitrary representations. As seen in the table, since $p$ and $q$ are nonnegative, the sign of the interaction is determined for a given $\alpha$, except for $[p+2, q-1]$ and $[p-1, q+2]$. The interactions of these channels are either attractive or repulsive, depending on the values of $p$ and $q$. It is worth noting that $C_{\alpha, T}$ is an integer, since $p$ and $q$ are also integers.

In the general expression (12), it is not known which representations are exotic before specifying the baryon number of the target hadron. In order to discuss the exotic states, we define the exoticness $E[41,42,43,44]$ as the number of valence quark-antiquark pairs to compose the flavor multiplet. We denote $B$ as the baryon number carried by $u, d$, and $s$ quarks. The number of the heavy quarks is not counted as the baryon number here. For $B>0$, the exoticness $E$ is given by

$$
E=\epsilon \theta(\epsilon)+\nu \theta(\nu),
$$

where

$$
\epsilon \equiv \frac{p+2 q}{3}-B, \quad \nu \equiv \frac{p-q}{3}-B .
$$

Note that $\epsilon \geq \nu$. More general arguments and derivations are given in Appendix $\mathrm{A}$

We are interested in the channel $\alpha$ which has a larger exoticness $E$ than that of the target hadron $T$. To find it we define

$$
\Delta \epsilon=\epsilon_{\alpha}-\epsilon_{T}, \quad \Delta \nu=\nu_{\alpha}-\nu_{T},
$$

which are shown in the fifth and sixth columns of Table III. With these quantities, we evaluate the difference of the exoticness $\Delta E=E_{\alpha}-E_{T}$ :

$$
\Delta E= \begin{cases}0 & \text { for } \quad \epsilon_{\alpha} \leq 0, \epsilon_{T} \leq 0 \\ \Delta \epsilon & \text { for } \nu_{\alpha} \leq 0, \nu_{T} \leq 0 \\ \Delta \epsilon+\Delta \nu & \text { for } \quad \text { others }\end{cases}
$$

Therefore, the condition to generate the states with larger exoticness than the target $(\Delta E=1)$ is given by one of the following cases:

(i) $\Delta \epsilon=1, \Delta \nu=0, \epsilon_{T} \geq 0$,

(ii) $\Delta \epsilon=0, \Delta \nu=1, \nu_{T} \geq 0$,

(iii) $\Delta \epsilon=1, \Delta \nu=-1, \nu_{T} \leq 0$.

These cases correspond to the first three rows of TableIII For the target in $[p, q]$, case (i) is satisfied for $\alpha=[p+$ $1, q+1]$, but the interaction is always repulsive for this channel. Case (ii) is satisfied for $\alpha=[p+2, q-1]$. In order to have attraction, however, $p=0$ is required, which leads to $B \leq-q / 3$ because of $\nu_{T} \geq 0$. This is achieved by the hadrons with negative baryon number, which are not considered here. Case (iii) is satisfied for $\alpha=[p-1, q+2]$, where the interaction can be attractive only when $q=0$ and the strength is $C_{\alpha, T}=1$. In this case, the condition $\nu_{T} \leq 0$ gives $p \geq 3 B$.

What we have shown here is that the attractive interaction in more "exotic" channel than the target hadron is only found as

$$
C_{\text {exotic }}=1
$$

with

$$
T=[p, 0], \quad \alpha=[p-1,2], \quad p \geq 3 B .
$$

It is interesting that the strength is always $C_{\alpha, T}=1$, which is the smallest strength of the Weinberg-Tomozawa term. In addition, this takes place when the target hadron belongs to the totally symmetric representation $[p, 0]$. The examples shown above for the ground states 
are the special cases of this conclusion with $p=1,2$, and $3(T=\mathbf{3}, \mathbf{6}$, and $\mathbf{1 0}$, respectively).

Let us consider the exoticness of the representation $T=[p, 0]$ with $p \geq 3 B$,

$$
E=\left(\frac{p}{3}-B\right)+\left(\frac{p}{3}-B\right)=2\left(\frac{p}{3}-B\right) .
$$

It follows from the condition of triality A1 that $(p / 3-$ $B)$ is an integer. This means that the attractive channels to generate more exotic states appear for even number of $E$. For instance, for $B=1$ hadrons, $[3,0]$ is $E=0$ and $[6,0]$ is $E=2$, and there is no such channel in $E=1$. Thus, even if the attraction (14) is enough to generate a bound state, the WT interaction can generate at most $\Delta E=+1$ state, and it is not possible to generate a tower of exotic states recursively.

The next question is whether the attraction (14) is strong enough to provide a bound state. This will be discussed in Sec. IV]

\section{Large- $N_{c}$ limit of the WT term}

In this section we discuss the large- $N_{c}$ limit of the WT term. It is known that the WT term scales as $\mathcal{O}\left(1 / N_{c}\right)$ in the large- $N_{c}$ limit, since it contains $1 / f^{2}$ and $f \sim \mathcal{O}\left(\sqrt{N_{c}}\right)$ [45, 46]. However, if the coupling strength $C_{\alpha, T}$ has some $N_{c}$ dependence, the scaling of the amplitude will be different. In Ref. [47], nontrivial $N_{c}$ dependence of the WT term was reported for the spin-flavor $\mathrm{SU}(6)$ extended WT term. Here we show that $C_{\alpha, T}$ does have the $N_{c}$ dependence in the case of the baryon target without incorporating the spin degrees of freedom. The representations of mesons do not depend on $N_{c}$ so that the result for the heavy meson target remains unchanged. An interesting discussion was recently made on interplay of the chiral and $1 / N_{c}$ expansions [48]. In the present argument, we perform the chiral expansion first, then consider the large- $N_{c}$ expansion.

For arbitrary $N_{c}$, a baryon is constructed by $N_{c}$ quarks and $E$ pairs of quarks and antiquarks, where $E$ is the exoticness. Accordingly, the SU(3) flavor representations for the baryon are extended as [49, 50]

$$
[p, q] \rightarrow\left[p, q+\frac{N_{c}-3}{2}\right]
$$

which reduces to $[p, q]$ at $N_{c}=3$. In this extension, the baryon spin is implicitly fixed at the value of $N_{c}=3$. Denoting the $N_{c}$ extended representation of $\boldsymbol{R}$ as " $\boldsymbol{R}$ ", we find that the coupling strengths $C$ " $\alpha$ ", " $T$ " with arbitrary $N_{c}$ can be given by

$$
C " \alpha ", " T "=C_{2}(" T ")-C_{2}(" \alpha ")+3 .
$$

Note that the representation of the meson does not change in the large- $N_{c}$ limit. The general form of the quadratic Casimir is given in Eq.(10). The Casimir of the large- $N_{c}$ baryon " $[p, q]$ " is given by

$$
\begin{aligned}
C_{2}("[p, q] ")= & C_{2}\left(\left[p, q+\frac{N_{c}-3}{2}\right]\right) \\
= & \frac{1}{3}\left(-\frac{9}{4}+p^{2}+\frac{3 p}{2}+p q+q^{2}\right) \\
& +\frac{1}{3}\left(\frac{p}{2}+q\right) N_{c}+\frac{N_{c}^{2}}{12},
\end{aligned}
$$

for arbitrary $N_{c}$. Note that $C_{2}("[p, q] ") \neq C_{2}("[q, p] ")$, because " $[p, q] " \neq \overline{"[q, p] "}$ for $N_{c} \neq 3$ by the construction of the large- $N_{c}$ baryon (15). It is important to note also that the coefficient of the linear term of $N_{c}$ in the righthand side of Eq. (17) depends on the representation $[p, q]$, while the coefficient of $N_{c}^{2}$ is independent of the representation. This means that there is nontrivial linear $N_{c}$ dependence at order $\mathcal{O}\left(N_{c}\right)$ in the coefficient of the WT term: $C$ " $\alpha$ " " $T$ " $\left(N_{c}\right)=C_{2}(" T ")-C_{2}(" \alpha ")+3$, where the leading $N_{c}^{2}$ terms are canceled. This is consistent with the general $N_{c}$ dependence $\mathcal{O}\left(N_{c}^{0}\right)$ of the meson-baryon interaction.

For specific target hadrons, using Eqs. (16) and (17) we evaluate the $C$ " $\alpha$ ", " $T$ " $\left(N_{c}\right)$ coefficients with arbitrary $N_{c}$. These are summarized in TableIV for light flavor baryons and in Table $\mathrm{V}$ for heavy flavor hadrons. It is worth noting that the attraction of $C$ "27", "10" with $N_{c}=3$ turns into repulsion for $N_{c}>5$, which is also the case for $C$ " $\overline{\mathbf{1 5}}$ ", "6". Thus, the attractions found in exotic baryon channels with $N_{c}=3$ change into repulsive interaction at large $N_{c}$. Moreover, the repulsion in the exotic channels linearly increases in the large- $N_{c}$ limit. It is also interesting that the attraction in the coupling C "1", "8" linearly increases as $N_{c}$ is increased. As mentioned above, the heavy meson $q \bar{Q}$ does not change its representation in the large- $N_{c}$ limit. Therefore the strengths shown in Table II hold in arbitrary $N_{c}$, and the exotic channel $\alpha=\overline{\mathbf{6}}$ remains attractive, as shown in Table $\mathrm{V}$.

The above discussion is on the large- $N_{c}$ behavior of the coupling strengths. Recalling that the WT term has $N_{c}$ dependence of $1 / f^{2}$, the scaling of the amplitude of

TABLE IV: The coupling strengths $C$ " $\alpha$ ", "T" $\left(N_{c}\right)$ for light fla-

\begin{tabular}{|c|c|c|c|c|c|c|c|}
\hline$\alpha$ & " $\overline{3} "$ & "6" & $" \overline{15} "$ & "24" & 3 & $\overline{6}$ & 15 \\
\hline$T=" \overline{3} "$ & 3 & 1 & $\frac{1}{2}-\frac{N_{c}}{2}$ & & & & \\
\hline $\begin{array}{c}T=" 6 " \\
T=\mathbf{3}\end{array}$ & 5 & 3 & $\frac{5}{2}-\frac{N_{c}}{2}$ & $-\frac{1}{2}-\frac{N_{c}}{2}$ & 3 & 1 & -1 \\
\hline
\end{tabular}
vor baryons.

\begin{tabular}{ccccccc}
\hline \hline$\alpha$ & "1" & "8" & "10" & "10" & "27" & "35" \\
\hline$T=" 8 "$ & $\frac{9}{2}+\frac{N_{c}}{2}$ & 3 & 0 & $\frac{3}{2}-\frac{N_{c}}{2}$ & $-\frac{1}{2}-\frac{N_{c}}{2}$ & \\
$T=" 10 "$ & & 6 & 3 & & $\frac{5}{2}-\frac{N_{c}}{2}$ & $-\frac{3}{2}-\frac{N_{c}}{2}$ \\
\hline \hline
\end{tabular}

TABLE V: The coupling strengths $C$ " $\alpha$ ", " $T$ " $\left(N_{c}\right)$ for heavy flavor hadrons. $T=\mathbf{3}$ is assigned to heavy mesons so that $C_{\alpha, T}\left(N_{c}\right)$ are unchanged. 
the WT term is in total given by the product of $1 / N_{c}$ and $C$ " $\alpha ", " T "\left(N_{c}\right)$. Therefore, in large- $N_{c}$ limit, the WT interaction

(i) is attractive for the " 1 " channel in the scattering of the " 8 " baryon and the NG boson,

(ii) has no interaction for other nonexotic baryonic channels,

(iii) is repulsive for all exotic baryonic channels,

(iv) has no interaction for nonexotic and exotic mesonic channels.

The first statement is very interesting in the context of the two-pole structure of $\Lambda(1405)$ [14, 15, 16]. It was found that two poles originate in the singlet and octet bound states in the $\mathrm{SU}(3)$ limit 14]. Although the SU(3) breaking causes admixture of the singlet and octet states, different behavior of the poles is expected in the large$N_{c}$ limit. The investigation of the large- $N_{c}$ limit would provide deeper understanding of the $\Lambda(1405)$ resonance.

We show that the coupling in the " 1 " channel is attractive in the large- $N_{c}$ limit, while " $\overline{\mathbf{1 0}} "$, "27", and "35" channels are all repulsive. This observation may be related to the kaon bound state approach to the Skyrmion [51] which is essentially based on the large- $N_{c}$ limit. In this approach, $s$-wave bound states of $K$ with the $\mathrm{SU}(2)$ soliton are obtained for $S=-1$ channel, which corresponds to $\Lambda(1405)$ and may largely have the flavor singlet component. On the other hand, exotic $S=+1$ states are not bound [52, 53]. The relation between two different approaches in the large- $N_{c}$ limit is worth investigating, although the present analysis based on the $\mathrm{SU}(3)$ symmetric limit, while the bound state approach is based on the large $\mathrm{SU}(3)$ breaking.

We can also extend the large- $N_{c}$ limit to the general target hadrons belonging to the representation $[p, q]$. In the seventh column of Table III, we show the coupling strength at arbitrary $N_{c}$. Combining with the $1 / N_{c}$ factor of $1 / f^{2}$, the sign of the WT interaction at the large- $N_{c}$ limit can be obtained in the eighth column of TableIII It is seen that in the large- $N_{c}$ limit, among the exotic channels, $\alpha=[p+1, q+1]$ and $\alpha=[p-1, q+2]$ are repulsive and the strength of the interaction for $\alpha=[p-1, q+2]$ is zero. In this way, we show that no attractive interaction exists for exotic channels in the large- $N_{c}$ limit. This means that, in the large- $N_{c}$ limit, the nonexistence of the $s$-wave exotic baryons can be shown without solving the scattering problem.

\section{BOUND STATE SOLUTIONS}

\section{A. Condition to generate a bound state}

We have been discussing the kernel interaction of the chiral unitary approach so far and have found the possible attractive interaction in exotic channels with uni- versal strength $C_{\text {exotic }}=1$. Now we study the unitarized amplitude (3) with the WT interaction, and derive the condition to generate a bound state pole. In other words, we solve the Schrödinger equation with the potential (2). Before studying the chiral unitary approach, let us note that the WT interaction is independent of the three-momentum, as seen in Eq. (2). Therefore it is instructive to recall an analogous problem of the delta function potential in three-dimensional nonrelativistic quantum mechanics.

As is well known, in one spatial dimension, the delta function potential always provides one bound state, if the interaction is attractive. However, in three dimensions, the existence of a bound state is not trivial. Moreover, an ultraviolet divergence appears in obtaining the wave function in coordinate space, as we show in Appendix B. This is because the eigenvalue problem is ill defined, since the short distance behavior of the potential is more singular than the kinetic term [54]. To obtain a physically meaningful result, we should tame the divergence by a proper regularization scheme. With a three-momentum cutoff $\Lambda$, the binding energy of a bound state $E_{b}$ in the three-dimensional delta function potential $V(\boldsymbol{x})=-v \delta(\boldsymbol{x})$ is given by the equation

$$
\frac{1}{2 m v}=\frac{1}{2 \pi^{2}}\left(\Lambda-\sqrt{2 m E_{b}} \arctan \left[\frac{\Lambda}{\sqrt{2 m E_{b}}}\right]\right),
$$

where $m$ is the reduced mass of the system (see Appendix (B). Note that the binding energy depends on the cutoff $\Lambda$, and the solution does not always exist. The condition to have a bound state solution is

$$
v>\frac{\pi^{2}}{m \Lambda} \equiv v_{c},
$$

where we have defined the critical strength of attraction $v_{c}$. For a given cutoff $\Lambda, v_{c}$ is the smallest attraction that can provide the bound state. If the attraction is less than $v_{c}$, no bound state exists. In addition, as shown in Appendix B, no resonance solution with a complex energy is found in the delta function potential.

Turning to the chiral unitary approach, the problem is quite similar to the delta function potential problem, but with the following differences: (i) relativistic kinematics and (ii) energy dependence of the coupling. In order to find the bound state in the scattering amplitude (3), we write the denominator of the amplitude as

$$
D(\sqrt{s}) \equiv 1-V(\sqrt{s}) G(\sqrt{s}) .
$$

Then the mass of the bound state with its mass $M_{b}$ is obtained by

$$
D\left(M_{b}\right)=0, \quad M_{T}<M_{b}<M_{T}+m .
$$

The mass of the bound state should be between the target mass $M_{T}$ and the scattering threshold $m+M_{T}$. Let us consider the behavior of the $D(\sqrt{s})$ in this region. It follows from Eq. (2) that $V\left(M_{T}\right)=0$, and in the present 
renormalization condition ([6), $G$ also vanishes at $\sqrt{s}=$ $M_{T}$, so that

$$
D\left(M_{T}\right)=1 .
$$

From Eqs. (2) and (44), it is seen that, for the attractive interaction $C_{\alpha, T}>0$, both $V(\sqrt{s})$ and $G(\sqrt{s})$ are monotonically decreasing in the region $M_{T}<\sqrt{s}<M_{T}+m$. Since $V\left(M_{T}\right)=G\left(M_{T}\right)=0, V G$ is positive and monotonically increasing for $M_{T}<\sqrt{s}<M_{T}+m$, and therefore we find that $D=1-V G$ is monotonically decreasing in this region. This means that there is only one bound state, if it exists, and the condition to satisfy Eq. (19) is given by $D\left(M_{T}+m\right)<0$. Thus, we define the critical strength of attraction $C_{\text {crit }}$ such that

$$
D\left(M_{T}+m\right)=0 .
$$

If the coupling strength is smaller than $C_{\text {crit }}$, no bound state exists. Substituting Eq. (22) into Eq. (20), we obtain

$$
C_{\text {crit }}=\frac{2 f^{2}}{m\left[-G\left(M_{T}+m\right)\right]},
$$

where

$$
G\left(M_{T}+m\right)=\frac{2 M_{T}}{(4 \pi)^{2}}\left[a\left(M_{T}\right)+\frac{m}{m+M_{T}} \ln \frac{m^{2}}{M_{T}^{2}}\right],
$$

with $\bar{q}=0$ at the threshold and $a\left(M_{T}\right)$ defined in Eq. (77). Note that $C_{\text {crit }}$ is a function of the mass of the target hadron $M_{T}$ once the mass $m$ and the decay constant $f$ of the NG boson are fixed.

It is worth noting that $V$ changes the sign at $\sqrt{s}=M_{T}$. The attractive WT interaction turns into a repulsive one for $\sqrt{s}<M_{T}$. This energy region corresponds to the kinematics of the (bound region of) $u$-channel scattering, which is not considered in the present formulation, since it is an unphysical region of the $s$-channel scattering. To study the amplitude of this region properly, we should include the $u$-channel multiscattering diagrams in the scattering equation or introduce the effect from the left-hand cut in the N/D method.

\section{B. Bound state spectrum}

Let us evaluate numerically the function $D(\sqrt{s})$ defined in Eq. (18), in order to find the energy of the bound state $M_{b}$ by

$$
D\left(M_{b}\right)=0 .
$$

For the numerical computation, we use the decay constant $f=93 \mathrm{MeV}$ and the mass of the NG bosons $m=368 \mathrm{MeV}$ which is the averaged value over the pseudoscalar octet mesons. We first choose the target hadron masses $M_{T}$ by averaging over the masses of the experimentally known ground states of the light flavor baryons,

\begin{tabular}{|c|c|c|c|c|c|c|}
\hline Target hadron & $T$ & $M_{T}(\mathrm{MeV})$ & $\alpha$ & $C_{\alpha, T}$ & $M_{b}(\mathrm{MeV})$ & $E_{b}(\mathrm{MeV})$ \\
\hline \multirow[t]{5}{*}{ Light baryon } & 8 & 1151 & $\overline{1}$ & 6 & 1450 & 69 \\
\hline & & & 8 & 3 & 1513 & 7 \\
\hline & 10 & 1382 & 8 & 6 & 1668 & 80 \\
\hline & & & 10 & 3 & 1737 & 13 \\
\hline & & & 27 & 1 & No solution & \\
\hline \multirow[t]{5}{*}{ Charmed baryon } & $\overline{3}$ & 2408 & $\overline{3}$ & 3 & 2736 & 40 \\
\hline & & & 6 & 1 & No solution & \\
\hline & 6 & 2534 & $\overline{3}$ & 5 & 2804 & 98 \\
\hline & & & 6 & 3 & 2860 & 42 \\
\hline & & & $\overline{\mathbf{1 5}}$ & 1 & No solution & \\
\hline \multirow[t]{2}{*}{$D$ meson } & $\mathbf{3}_{c}$ & 1900 & 3 & 3 & 2240 & 28 \\
\hline & & & $\overline{6}$ & 1 & No solution & \\
\hline \multirow[t]{2}{*}{$B$ meson } & $\mathbf{3}_{b}$ & 5309 & 3 & 3 & 5600 & 77 \\
\hline & & & $\overline{6}$ & 1 & No solution & \\
\hline
\end{tabular}
the charmed baryons, and the $D$ and $B$ mesons given by
TABLE VI: Masses $M_{T}$ and coupling constants $C_{\alpha, T}$ for several targets in the $\mathrm{SU}(3)$ limit. The masses of bound states $M_{b}$ are obtained by solving Eq. (22) numerically.

Particle Data Group (PDG) [1]. These are presented in the third column of Table $\mathrm{VI}$.

In Fig. 2, we plot $D=1-V G$ for various target hadrons with the coupling strengths found in Sec. III A. The position where curves cross zero determine bound state energies. The resulting energies of bound states $M_{b}$ and the binding energies

$$
E_{b}=M_{T}+m-M_{b}
$$

are summarized in the sixth and seventh column of Table V1 As expected, larger coupling strengths provide larger binding energies. Phenomenology of the bound states found here has been extensively studied in more realistic calculations with $\mathrm{SU}(3)$ breaking, and has been shown to reproduce experimentally observed resonances [18, 19, 20, 25, 32, 33, 34]. It is worth noting that no bound state is found for the flavor exotic channels. The attraction in these channels $C_{\alpha, T}=1$ is not enough to bind the two-body system for the physical masses of these hadrons. This point will be further studied in next subsection.

It is instructive to show the dependence of the binding energy on the target mass. In Fig. 3 we plot the binding energies $E_{b}=M_{T}+m-M_{b}$ as functions of the mass of target $M_{T}$, fixing the coupling strengths as $C_{\alpha, T}=6,5$, and $3 . E_{b}=0$ corresponds to the bound state exactly at the threshold, and $E_{b}=m$ corresponds to $M_{b}=M_{T}$. We find that the larger mass of the target provides larger binding energy. Note that $C_{\alpha, T}=1$ does not generate a bound state in this energy region $\sqrt{s}<6 \mathrm{GeV}$.

The spectrum of the bound states in the light flavor sector shows an interesting structure, when we compare it with that of the $\mathrm{SU}(6)$ quark model. Recall the ground states and first excited states in the $\mathrm{SU}(6)$ quark model, which are $\mathbf{5 6}$ and $\mathbf{7 0}$, respectively. These representations include the spin-flavor quantum numbers of $\left\{{ }^{2 S+1} D_{\mathrm{SU}(3)}={ }^{2} \mathbf{8},{ }^{4} \mathbf{1 0}\right\}$ in $\mathbf{5 6}$, and $\left\{{ }^{2} \mathbf{1},{ }^{2} \mathbf{8},{ }^{2} \mathbf{8},{ }^{4} \mathbf{1},{ }^{4} \mathbf{8}\right\}$ in 70. In the chiral unitary approach, the bound states 

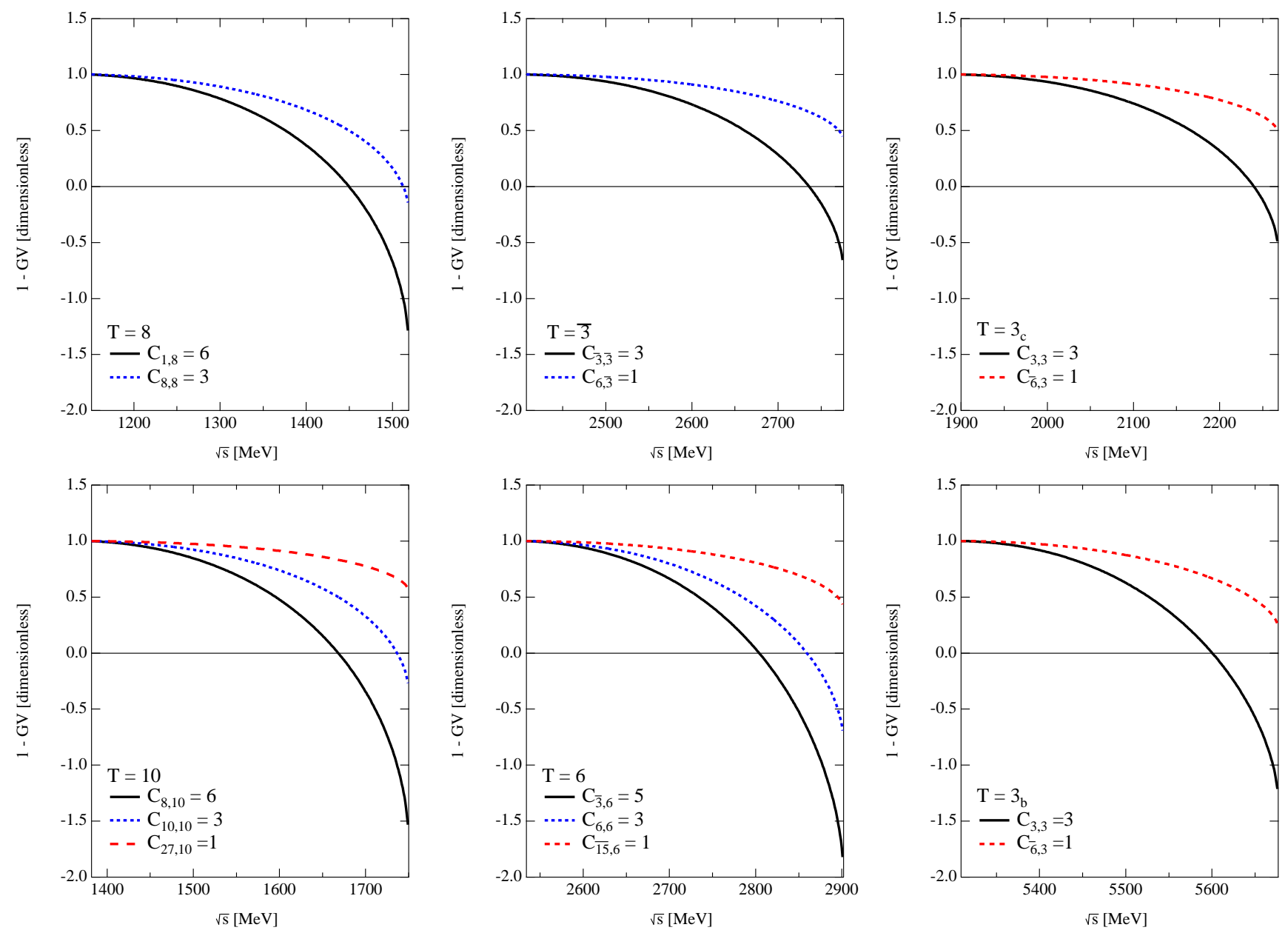

FIG. 2: (color online). The denominator of the scattering amplitude of $1-V G$ for light flavor baryons (top left panel: $T=\mathbf{8}$, bottom left panel: $T=\mathbf{1 0}$ ), for charmed baryon (top center panel: $T=\overline{\mathbf{3}}$, bottom center panel: $T=\mathbf{6}$ ) and for $D$ and $B$ mesons (top right panel: $T=\mathbf{3}_{c}$, bottom right panel: $T=\mathbf{3}_{b}$ ). Results for exotic channels are denoted by dashed lines.

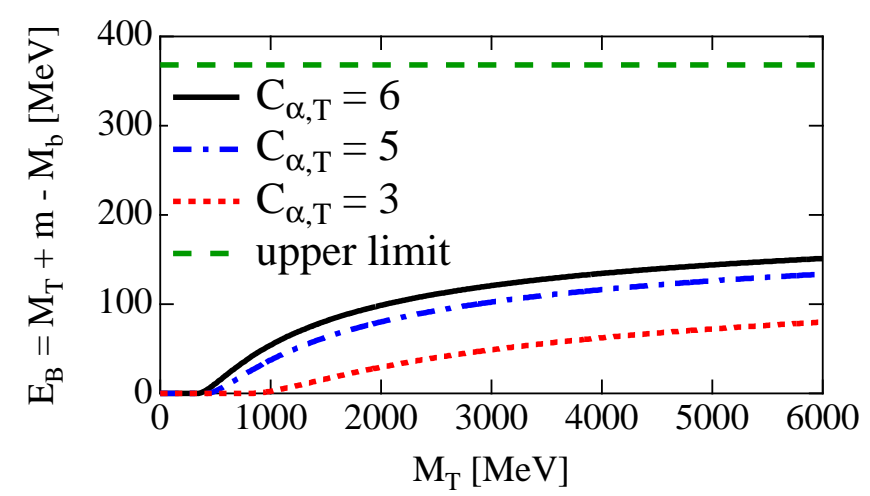

FIG. 3: (color online). Binding energies $E_{b}=M_{T}+m-M_{b}$ as functions of $M_{T}$ with coupling strengths $C_{\alpha, T}=6$ (solid line), 5 (dash-dotted line), and 3 (dotted line). For reference, the upper limit of the binding energies is indicated as the dashed line. No bound state is found for $C_{\alpha, T}=1$ in this energy region. of the baryon and NG boson have the same spin as the target baryon with the opposite parity, since the pseudoscalar NG boson is bound in the $s$-wave interaction. Let us take the ground state octet ${ }^{2} \mathbf{8}$ and decuplet ${ }^{4} \mathbf{1 0}$ as the target baryons. Then the spin-flavor quantum numbers of the hadron-NG boson bound states are ${ }^{2} \mathbf{1},{ }^{2} \boldsymbol{8},{ }^{2} \boldsymbol{8}$ from the octet target and ${ }^{4} \mathbf{8},{ }^{4} \mathbf{1 0}$ from the decuplet target, as shown in Table VI. Comparing the bound states obtained in the chiral unitary approach and the first excited states belonging to $\mathbf{7 0}$ in the quark model, we find that the ${ }^{4} \mathbf{1}$ bound state is absent in our approach but the ${ }^{4} \mathbf{1 0}$ state is present instead. It is interesting to see that, in the quark models, the ${ }^{4} \mathbf{1}$ state is assigned as $\Lambda(1520)$ [55], while in the chiral unitary approach it is reproduced as a ${ }^{4} \mathbf{8}$ dominant state [18, 56]. Therefore, examination of the property of the $\Lambda(1520)$ resonance will provide further understanding of the baryon spectroscopy, for instance, through the coupling to the vector mesons [57]. 


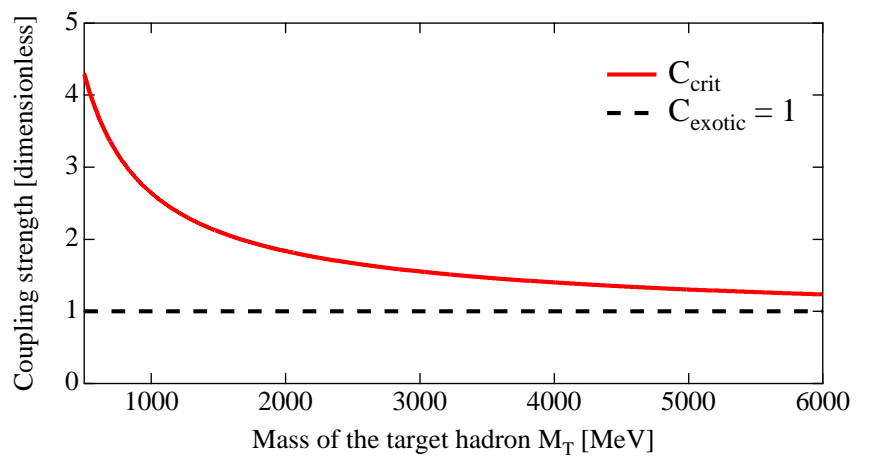

FIG. 4: (color online). Critical coupling strength $C_{\text {crit }}$ for $m=368 \mathrm{MeV}$ (solid line). The universal strength of the attraction in exotic channels $C_{\text {exotic }}=1$ is shown by the dashed line.

\section{Critical coupling strength}

As studied in Sec. IVA, we find the critical attractive strength $C_{\text {crit }}$ to generate a bound state. We plot $C_{\text {crit }}$ which is evaluated by Eq. (21) as a function of $M_{T}$ in Fig. 4, where we employ the meson decay constant $f=93 \mathrm{MeV}$ and the meson mass $m=368 \mathrm{MeV}$ which corresponds to the averaged mass of the octet mesons $(\pi$, $K, \eta)$. We also plot $C_{\text {exotic }}=1$, which is the universal strength of the possible attraction in exotic channels. It is clear that the attraction $C_{\text {exotic }}=1$ is not enough to bind the two-body system for the target mass $M<6$ $\mathrm{GeV}$, where all the hadronic target states we consider lie.

Let us examine the robustness of the conclusion. As seen in Fig. 4 the critical coupling $C_{\text {crit }}$ is monotonically decreasing as we increase $M_{T}$. Therefore it will become smaller than $C_{\alpha, T}=1$ at sufficiently large $M_{T}$. Quantitatively, in order to have a bound state for the exotic channel with $C_{\text {exotic }}=1$, the mass of the target hadron $M_{T}$ should be larger than about $14 \mathrm{GeV}$ for $m=368$ $\mathrm{MeV}$ and $f=93 \mathrm{MeV}$. Exotic hadrons can exist as bound states of the NG boson-hadron system if a stable hadron (in 3 representation, for instance) exists in this energy region. So far no hadronic states have been reported.

Next we study the dependence of $C_{\text {crit }}$ on $m$ and $f$. It follows from Eq. (21) that, as the decay constant $f$ is increased, the critical coupling $C_{\text {crit }}$ also increases. The dependence on $m$ is essentially determined by the prefactor $1 / m$ and the critical coupling $C_{\text {crit }}$ becomes smaller as we increase $m$, since the dependence of $G$ on $m$ is not so strong. In Fig. 5, we show the $C_{\text {crit }}=C_{\text {exotic }}$ lines in $m-f$ plane with three different values of $M_{T}=1000,3000$, and $6000 \mathrm{MeV}$. A parameter choice below the $C_{\text {crit }}=C_{\text {exotic }}$ lines provides $C_{\text {crit }}<1$, so that the exotic states can be bound. Above these liens, the attractive interaction in the exotic channels is not strong enough to generate a bound state.

In Fig. 5, we indicate the point $(m, f)=(368,93) \mathrm{MeV}$

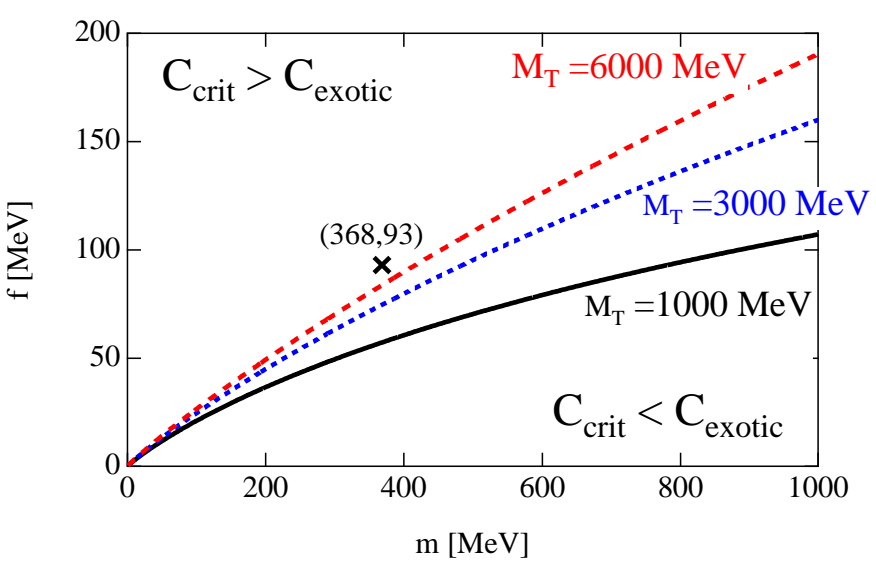

FIG. 5: (color online). Lines for $C_{\text {crit }}=1$ in $(m, f)$ plane, with $M_{T}=1000 \mathrm{MeV}$ (solid line), $M_{T}=3000 \mathrm{MeV}$ (dotted line), and $M_{T}=6000 \mathrm{MeV}$ (dashed line). In the region below (above) the lines, $C_{\text {crit }}<1\left(C_{\text {crit }}>1\right)$. The point of $(m, f)=$ $(368,93) \mathrm{MeV}$ is indicated by the cross.

by the cross. From this figure, we see that the NG boson could be bound in the exotic channel, if we use a heavier mass for the NG boson. For instance, with $m=500 \mathrm{MeV}$ an exotic bound state appears with $M_{T} \simeq 2500 \mathrm{MeV}$ as shown in Ref. [19]. One should however note that, to utilize the low energy theorem of the chiral symmetry, the mass of the NG boson should not be large. On the other hand, for a smaller NG boson mass, the critical strength $C_{\text {crit }}$ becomes larger. Indeed, with $m=m_{\pi}$, the critical strength $C_{\text {crit }} \sim 10$ for the mass of $N$ and $\Delta$. Using Eq. (11), we can show that the strongest attraction is $C_{1 / 2,1 / 2}=2$ for $T=N$ and $C_{1 / 2,3 / 2}=5$ for $T=$ $\Delta$. Therefore, attractions found in SU(2) meson-baryon scatterings do not generate the bound states, which is consistent with experimental observation.

In summary, we show that the critical coupling strength $C_{\text {crit }}$ is larger than the attractive interaction found in exotic channels, with the physically known values of parameters $M_{T}, m$, and $f$. The critical value $C_{\text {crit }}$ could be smaller than $C_{\text {exotic }}$ if the mass of the target hadron $M_{T}$ is sufficiently heavy, or the mass of the NG boson $m$ is large, or the decay constant of the NG boson $f$ is small.

\section{SUMMARY AND DISCUSSIONS}

In this paper, we have studied the $s$-wave bound states in the NG boson-hadron scattering described by the chiral unitary approach in flavor $\mathrm{SU}(3)$ limit. We have studied the group theoretical structure of the WeinbergTomozawa interaction, which is independent thanks to the chiral symmetry. The general expression of the coupling strengths of the WT interaction $C_{\alpha, T}$ have been derived. Considering the nonexotic hadrons as the target hadrons, we have found an attraction in several ex- 
otic channels with the strength $C_{\text {exotic }}=1$. More generally, based on the group theoretical argument, we have shown that the coupling strengths of the attractive interaction for the channel raising the exoticness are universally $C_{\text {exotic }}=1$, which is the smallest value in the WT interaction.

We have proved that the weak attraction $C_{\text {exotic }}=1$ of the exotic channel in the WT term is not enough to generate the bound state of the experimentally observed hadrons and the NG boson. For proof, we have derived the critical strength of attraction to generate a bound state $C_{\text {crit }}$, based on the general principles of the scattering theory. The critical strength is expressed in terms of the mass of the target hadron and the mass and decay constant of the NG boson. Studying the dependence of the critical strength on these parameters, we find that the exotic bound state could be generated by the WT term for larger $M_{T}$, larger $m$, and smaller $f$. We find, however, that these parameters for experimentally known hadrons do not satisfy $C_{\text {crit }}<C_{\text {exotic }}$. In other words, the strength of attraction in the exotic channels is very weak and smaller than the critical value, for the experimentally known particles.

We have also examined the large- $N_{c}$ behavior of the coupling strengths, which shows the nontrivial $N_{c}$ dependence. Because of the $N_{c}$ dependence of the coupling strengths, all amplitudes calculated by the WT term for the exotic channels become repulsive or vanish in the large- $N_{c}$ limit, including attractive ones at $N_{c}=3$. The analysis of the critical coupling, together with the large$N_{c}$ behavior of the WT interaction, shows the difficulty of generating the exotic state by the chiral interaction.

In the present approach, exotic hadrons are treated as quasibound states of the NG boson and a target hadron on the same footing with the nonexotic resonances. We have shown that the attractive interaction of the WT term is not enough to bind the system. Considering the fact that a certain number of known resonances have been properly generated by the chiral unitary approaches, our conclusion on the exotic states should be of great relevance. It should be noted, however, that the present analysis does not exclude the existence of the exotic states formed by other mechanisms, for instance the genuine quark states, because the WT interaction is one specific mechanism to generate states dynamically.

Apart from the existence of the genuine quark state, one may be cautious of the following uncertainties in the analysis; the flavor $\mathrm{SU}(3)$ symmetry is badly broken in nature, where it is known that the substantial breaking effect is of about $20 \%$. We implicitly assumed that the target particle is stable, although several states such as those in $\mathbf{1 0}$ can decay via the strong interactions. In recent studies of the chiral unitary approach [58, 59, 60, 61, 62], an important role played by the higher order terms of the chiral Lagrangian has been discussed, while we only take into account the leading order term. The channels which are zero or repulsive at the leading order can become attractive when the higher order terms are included. Indeed, some resonances can be generated by the effect of the higher order terms, which are absent in the leading order calculations [61].

Despite these caveats, the present formulation in the $\mathrm{SU}(3)$ limit is sufficient to discuss the qualitative features of the dynamically generated states. For instance, two poles corresponding to the $\Lambda(1405)$ resonance are found with and without the $\mathrm{SU}(3)$ breaking effects or the higher order terms [14, 29, 59, 62]. This fact indicates that the qualitative feature of the result is independent of the detailed construction of the model, and that the essential structure is determined by the WT term, which is common for all approaches. To perform a more quantitative analysis, corrections from the above-mentioned effects should be taken into account. This can be performed systematically, for instance, based on the chiral perturbation theory. For future perspective, it is important to include the $\mathrm{SU}(3)$ breaking effects to draw more quantitative conclusion. The bound states in the $\mathrm{SU}(3)$ limit were found to become resonances when the $\mathrm{SU}(3)$ breaking effect was taken into account. The mechanism for how the bound states acquire the width should be clarified, in order to connect the results in the $\mathrm{SU}(3)$ limit to the physical world. These will be summarized elsewhere.

\section{Acknowledgments}

The authors are grateful to Professor M. Oka for helpful discussions. We also thank Professor V. Kopeliovich for useful comments on exoticness. T. H. thanks the Japan Society for the Promotion of Science (JSPS) for financial support. This work is supported in part by the Grant for Scientific Research (No. 17959600, No. 18042001, and No. 16540252) and by Grant-in-Aid for the 21st Century COE "Center for Diversity and Universality in Physics" from the Ministry of Education, Culture, Sports, Science and Technology (MEXT) of Japan.

\section{APPENDIX A: EXOTICNESS}

In this section, we derive the exoticness quantum number $E$ [41, 42, 43, 44]. The exoticness expresses the minimal number of the valence quark-antiquark pairs to compose the flavor multiplet $[p, q]$ with a given baryon number $B$. The baryon number $B$ here counts only the $u, d$, and $s$ quarks, so that $B$ can be fractional for the hadrons with the heavy quarks. Here we obtain the formula of $E$ for arbitrary $B$ and $[p, q]$ for the first time.

Let us consider a hadron with the baryon number $B$ belonging to the $[p, q]$ representation in the flavor $\mathrm{SU}(3)$ group, where $p$ and $q$ are nonnegative integers. From the triality of the $[p, q]$ representation, $p+2 q$ is congruent to $3 B$ modulo 3 :

$$
p+2 q \equiv 3 B \quad(\bmod 3)
$$


This ensures that the exoticness quantum numbers are integers. We denote the numbers of the light valence quarks and antiquarks in the hadron as $n_{q}$ and $n_{\bar{q}}$, respectively. Then the baryon number and the exoticness are given by

$$
\begin{aligned}
B & =\frac{n_{q}-n_{\bar{q}}}{3}, \\
E & =\min \left(n_{q}, n_{\bar{q}}\right) .
\end{aligned}
$$

The exoticness is given by the antiquark number for the hadron with the positive baryon number $\left(n_{q}>n_{\bar{q}}\right)$ and the quark number for the negative baryon number $\left(n_{q}<\right.$ $\left.n_{\bar{q}}\right)$. We have an exception for the light mesons $(B=0)$. Their exoticness is given by

$$
E=n_{q}-1=n_{\bar{q}}-1 \text { for light meson. }
$$

The valence quark contents $\left(n_{q}, n_{\bar{q}}\right)$ of the hadron are uniquely determined by the baryon number $B$ and the representation $[p, q]$. Therefore, once we know the quark contents $\left(n_{q}, n_{\bar{q}}\right)$ of the hadron, we obtain the exoticness $E$ through Eqs. (A3) and (A4). In the following, we express $n_{q}$ and $n_{\bar{q}}$ in terms of $B, p$, and $q$.

Let us consider the Young tableau of the representation $[p, q]$, which is expressed diagrammatically by

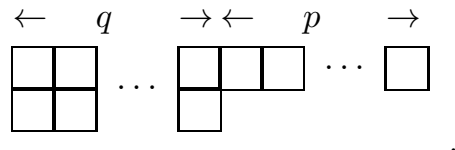

In order to know the valence quark contents of the hadron with $B$ and $[p, q]$, we assign these boxes to quarks and antiquarks so as to give the baryon number $B$ of the hadron. the quark is denoted by one box, while the antiquark is expressed by two boxes in an antisymmetric combination (two boxes in different rows). The baryon number which can be expressed by the boxes is limited within the following range:

$$
\beta_{-} \leq B \leq \beta_{+}
$$

with

$$
\beta_{-} \equiv \frac{p-q}{3}, \quad \beta_{+} \equiv \frac{p+2 q}{3}
$$

The lower limit $\beta_{-}$is given by maximizing the number of antiquarks, while the upper limit $\beta_{+}$corresponds to the assignment of all the boxes for quarks. There are two cases: (i) the present boxes are enough to obtain the baryon number, that is, the baryon number of the hadron is within the range (A5); (ii) some extra boxes with the flavor singlet combination are necessary to express the baryon number. The addition of the extra boxes with the flavor singlet combination can be done without changing the representation $[p, q]$.

For case (i), since the number of the boxes in the Young tableau is just enough to give the quark contents of the hadron, the total number of the boxes, $p+2 q$, is given by

$$
p+2 q=n_{q}+2 n_{\bar{q}} .
$$

Combining Eqs. (A2) and (A7), we obtain the quark contents in terms of $B, p$, and $q$ as

$$
n_{q}=\frac{p+2 q}{3}+2 B, \quad n_{\bar{q}}=\frac{p+2 q}{3}-B .
$$

For case (ii), the baryon number cannot be obtained from the existing boxes, so we add some sets of three boxes forming the flavor singlet (totally antisymmetric) combination with nonzero baryon number:

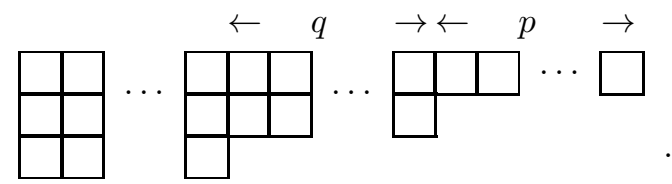

Note that the addition of the flavor singlet with zero baryon number is meaningless, since it supplies just sea quark pairs. An example of case (ii) is the flavor singlet hadron with $B=1$. Since the Young tableau of the flavor singlet $[0,0]$ has no box, the baryon number $B=1$ cannot be achieved by the present box. Thus we add one set of three boxes with the flavor singlet and assign quarks to these boxes to give $B=1$. Then we obtain the valence quark contents as $n_{q}=3$ and $n_{\bar{q}}=0$ for the flavor singlet hadron with $B=1$.

Case (ii) can be further classified into two cases:

$$
B<\beta_{-} \text {or } \beta_{+}<B
$$

For the case $\beta_{+}<B$, the baryon number $B$ exceeds the upper limit $\beta_{+}$. Recalling that $\beta_{+}$is obtained so as to assign all the boxes to quarks, that is, the quark number is $p+2 q$ and the antiquark number is zero, we see that the boxes for quarks are not enough to express the baryon number. To fill the deficit, we add $B-\beta_{+}$sets of three boxes in one column forming the flavor singlet and assign quarks to the boxes. The addition of one set of boxes changes the baryon number by +1 . Hence, we obtain

$$
n_{q}=p+2 q+3\left(B-\beta_{+}\right)=3 B, \quad n_{\bar{q}}=0 .
$$

For the case $B<\beta_{-}$, the baryon number $B$ is less than the lower limit $\beta_{-}$. In the lower limit, the antiquarks are maximally assigned to the boxes, that is, the quark number is $p$ and the antiquark number is $q$. Since $B<\beta_{-}$means a lack of boxes for antiquarks to express the baryon number, we add $\beta_{-}-B$ sets of the flavor singlet combination and maximally assign antiquarks to the whole boxes. The addition of $\beta_{-}-B$ sets of boxes changes the total number by -1 . Hence we obtain

$$
\begin{aligned}
& n_{q}=p-\left(\beta_{-}-B\right)=\beta_{+}+\beta_{-}+B, \\
& n_{\bar{q}}=q+2\left(\beta_{-}-B\right)=\beta_{+}+\beta_{-}-2 B .
\end{aligned}
$$


Summarizing all the cases, we obtain the valence quark contents of the hadron with the baryon number $B$ belonging to the $\mathrm{SU}(3)$ representation $[p, q]$ :

$$
\begin{aligned}
& n_{q}= \begin{cases}3 B & \text { for } \beta_{+} \leq B, \\
\beta_{+}+2 B & \text { for } \quad \beta_{-} \leq B \leq \beta_{+}, \\
\beta_{+}+\beta_{-}+B & \text { for } B \leq \beta_{-},\end{cases} \\
& n_{\bar{q}}=\left\{\begin{array}{lll}
0 & \text { for } \beta_{+} \leq B, \\
\beta_{+}-B & \text { for } \beta_{-} \leq B \leq \beta_{+}, \\
\beta_{+}+\beta_{-}-2 B & \text { for } B \leq \beta_{-},
\end{array}\right.
\end{aligned}
$$

where $\beta_{-}$and $\beta_{+}$are defined in Eq. (A6). Substituting Eqs. A9 and (A10) into Eqs. (A3) and (A4), we obtain the exoticness for the hadron with $B$ and $[p, q]$.

In the present study, we are interested in $B>0$. In this case, the exoticness $E=n_{\bar{q}}$ is given by

$$
E= \begin{cases}0 & \text { for } \quad \epsilon \leq 0 \\ \epsilon & \text { for } \epsilon \geq 0, \nu \leq 0 \\ \epsilon+\nu & \text { for } \quad \nu \geq 0\end{cases}
$$

with $\epsilon \equiv \beta_{+}-B$ and $\nu \equiv \beta_{-}-B$. This is equivalently expressed as

$$
E=\epsilon \theta(\epsilon)+\nu \theta(\nu),
$$

with the step function $\theta(x)$.

The exoticness quantum number was introduced in the context of the chiral soliton models [41, 42], but without considering the case (A8). The insufficiency of the definition was realized [43] and later the definition was generalized to give correct values for $B<\beta_{-}$[44], while the definition of $B>\beta_{+}$states was still not properly treated. This is enough for the states in the excited spectrum of soliton rotation, since there is no state with $B>\beta_{+}$. In our definition, the states with $B>\beta_{+}$can be correctly treated, and the generalization to the arbitrary baryon number is performed.

The exoticness derived above can be extended to the baryon with arbitrary $N_{c}$. The minimal contents of the baryon is $N_{c}$ quarks. The baryon number for a hadron with $n_{Q}$ heavy quarks is given by $B=1-n_{Q} / N_{c}$. From this and the triality of the flavor $\mathrm{SU}(3)$, we have, instead of Eqs. (A1) and (A2),

$$
p+2 q \equiv N_{c} B \quad(\bmod 3), \quad B=\frac{n_{q}-n_{\bar{q}}}{N_{c}} .
$$

Following the same argument as before with the replacement $B \rightarrow \frac{N_{c}}{3} B$, we obtain the numbers of quarks and antiquarks for arbitrary $N_{c}$,

$$
\begin{aligned}
& n_{q}= \begin{cases}N_{c} B & \text { for } \beta_{+} \leq \frac{N_{c}}{3} B, \\
\beta_{+}+\frac{2 N_{c}}{3} B & \text { for } \beta_{-} \leq \frac{N_{c}}{3} B \leq \beta_{+}, \\
\beta_{+}+\beta_{-}+\frac{N_{c}}{3} B & \text { for } \quad \frac{N_{c}}{3} B \leq \beta_{-},\end{cases} \\
& n_{\bar{q}}= \begin{cases}0 & \text { for } \beta_{+} \leq \frac{N_{c}}{3} B, \\
\beta_{+}-\frac{N_{c}}{3} B & \text { for } \beta_{-} \leq \frac{N_{c}}{3} B \leq \beta_{+}, \\
\beta_{+}+\beta_{-}-\frac{2 N_{c}}{3} B & \text { for } \frac{N_{c}}{3} B \leq \beta_{-} .\end{cases}
\end{aligned}
$$

From Eq. A3 , the exoticness of a baryon is given by $E=n_{\bar{q}}$.

\section{APPENDIX B: DELTA FUNCTION POTENTIAL}

Let us consider the $d$-dimensional delta function potential problem in quantum mechanics [54]. The Schrödinger equation takes on the form with $\hbar=1$

$$
-\frac{1}{2 m} \nabla^{2} \psi(\boldsymbol{r})+V(\boldsymbol{r}) \psi(\boldsymbol{r})=E \psi(\boldsymbol{r}),
$$

with the reduced mass of the system $m$ and the $d$ dimensional delta function potential $V(\boldsymbol{r})=-v \delta(\boldsymbol{r})$. We consider the attractive interaction $v>0$. To get an essential feature of the problem, it is convenient to consider it in a variational method. Let us take an expectation value of the Hamiltonian of the sum of the kinetic and potential terms with a normalized $s$-wave trial wave function with a size parameter, say $b$, as a variational parameter. Then the energy plot as a function of $b$ has one minimum for $d=1$, while it has no such a stable point for $d=3$; it falls into $-\infty$ as $b \rightarrow 0$, meaning that the delta function potential is too strongly attractive to overcome the kinetic energy due to the uncertainty principle.

For more quantitative discussions, we consider the wave function explicitly which is given in momentum space as

$$
\phi(\boldsymbol{p})=\frac{v_{0} \psi(\mathbf{0})}{p^{2}+\tilde{E}_{b}}
$$

where $v_{0}=2 m v$ and $\tilde{E}_{b}=2 m E_{b} \equiv-2 m E>0$. Integrating both sides over $\boldsymbol{p}$, we obtain

$$
\begin{aligned}
\frac{1}{v_{0}} & =\frac{1}{(2 \pi)^{d}} \int d^{d} \boldsymbol{p} \frac{1}{p^{2}+\tilde{E}_{b}} \\
& =\frac{1}{(2 \pi)^{d}} \int d \Omega \int d p p^{d-1} \frac{1}{p^{2}+\tilde{E}_{b}} .
\end{aligned}
$$

Here we use $\int d^{d} \boldsymbol{p} \phi(\boldsymbol{p})=\psi(\mathbf{0})$. Notice that the integral is divergent for $d \geq 2$ so that we need regularization. For $d=3$, by introducing the cutoff $\Lambda$ in the threemomentum, this integration leads to

$$
\frac{1}{v_{0}}=\frac{1}{2 \pi^{2}}\left(\Lambda-\sqrt{\tilde{E}_{b}} \arctan \left[\frac{\Lambda}{\sqrt{\tilde{E}_{b}}}\right]\right) .
$$

The energy of the bound state $E_{b}$ can be obtained by solving this equation, which depends not only on $v$ but also $\Lambda$. In addition, we need a condition in order to obtain the solution for Eq. (B1):

$$
v>\frac{\pi^{2}}{m \Lambda} \equiv v_{c} .
$$

This equation determines the critical strength of attraction $v_{c}$ that can provide the bound state, for a given 
cutoff $\Lambda$. If the attraction is weaker than $v_{c}$, no bound state appears.

For the scattering state, the wave function in momentum space $\phi(\boldsymbol{p})$ is given by

$$
\phi(\boldsymbol{p})=(2 \pi)^{3} \delta(\boldsymbol{p}-\boldsymbol{k})+\frac{v_{0}}{p^{2}-k^{2}-i \epsilon} \psi(\mathbf{0}),
$$

where $k^{2}=2 m E$. Integrating both sides over $\boldsymbol{p}$, we obtain the scattering amplitude $f(k)$ as

$$
\begin{aligned}
f(k) & =\frac{v_{0} \psi(\mathbf{0})}{2 \pi}=\frac{1}{2 \pi}\left(\frac{1}{v_{0}}-\frac{1}{2 \pi^{2}}\left[\Lambda+\frac{k}{2} \ln \frac{k-\Lambda}{k+\Lambda}\right]\right)^{-1} \\
& \equiv \frac{1}{2 \pi D(k)} .
\end{aligned}
$$

Here we use, for a complex value $k$,

$$
I=\int_{0}^{\Lambda} d p \frac{p^{2}}{p^{2}-k^{2}-i \epsilon}=\Lambda+\frac{k}{2} \ln \frac{k-\Lambda}{k+\Lambda} .
$$

This integral $I$ with the complex $k$ is obtained through analytic continuation of the integral with a real value $k$ :

$$
I=\left\{\begin{array}{ll}
\Lambda+\frac{k}{2}\left[\ln \left|\frac{\Lambda-k}{\Lambda+k}\right|+i \pi\right] & \text { for } \quad k<\Lambda \\
\Lambda+\frac{k}{2} \ln \left|\frac{\Lambda-k}{\Lambda+k}\right| & \text { for } \quad k>\Lambda
\end{array} .\right.
$$

Bound states and resonances appear when $f(k)$ has poles [or equivalently, $D(k)=0$ ] at

$$
\begin{array}{lll}
\operatorname{Re}[k]=0, & \operatorname{Im}[k]>0 & \text { for a bound state, } \\
\operatorname{Re}[k]>0, & \operatorname{Im}[k]<0 & \text { for a resonance state. }
\end{array}
$$

Indeed, the condition that $D(k)$ should be zero for pure imaginary $k$ is equivalent to Eq. (B1). In order to obtain a resonance state, the imaginary part of $D(k)$ should necessarily be zero with $\operatorname{Im}[k]<0$, which can be decomposed as

$\operatorname{Im}[D(k)]=-\frac{1}{2 \pi^{2}}\left(\frac{\operatorname{Re}[k]}{2} \arg \frac{k-\Lambda}{k+\Lambda}+\frac{\operatorname{Im}[k]}{2} \ln \left|\frac{k-\Lambda}{k+\Lambda}\right|\right)$.

Now the first term should be positive because the argument is defined in the range $0 \leq \theta<2 \pi$. Since $|k+\Lambda|>|k-\Lambda|$

$$
\ln \left|\frac{k-\Lambda}{k+\Lambda}\right|<0
$$

and therefore the second term is also positive. Thus we find $\operatorname{Im}[D(k)]<0$ for Eq. ( $(\overline{\mathrm{B} 2})$, and no resonance state appears.

\section{APPENDIX C: HEAVY MESON TARGET}

Here we address the formulation of the chiral unitary approach for the case with the meson target. The scattering amplitude is given in the same way as Eq. (3):

$$
t=V+V G t=\frac{1}{1-V G} V
$$

with the interaction $V$ and the loop function $G$. Denoting $V_{B}$ and $G_{B}$ for a baryon target equivalent to the expressions in Sec. II and $V_{M}$ and $G_{M}$ for a meson target given in Ref. [20], we have

$$
\begin{aligned}
V_{B} & =-\frac{1}{2 f^{2}} C \omega \\
V_{M} & =-\frac{1}{8 f^{2}} C\left(3 s-2 M_{T}^{2}-2 m^{2}-\frac{\left(M_{T}^{2}-m^{2}\right)^{2}}{s}\right),
\end{aligned}
$$

where $\sqrt{s}$ is the total energy and $M_{T}$ and $m$ are the masses of the target and the NG boson. We have used our convention for the coupling strength $C$. The loop function is given by

$$
\begin{aligned}
G_{B} & =i \int \frac{d^{4} q}{(2 \pi)^{4}} \frac{2 M_{T}}{(P-q)^{2}-M_{T}^{2}+i \epsilon} \frac{1}{q^{2}-m^{2}+i \epsilon}, \\
G_{M} & =\frac{G_{B}}{2 M_{T}} .
\end{aligned}
$$

Note that the dimensions of $G$ and $V$ in the two cases are different, reflecting the factor $2 M_{T}$ which comes from the normalization of the fermion spinor $\bar{u} u=1$.

We expand the interaction potentials in terms of $\omega / M_{T}$, where $\omega$ is the NG boson energy. Since we are interested in the low energy dynamics of the NG boson, the energy of the NG boson is of order of its mass. The target meson mass is taken to be large in comparison with the NG boson mass. Since $\sqrt{s}$ also depends on $M_{T}$, it is better to express the amplitudes as functions of the meson energy $\omega$ to take the large $M_{T}$ limit. Expanding the interaction $V_{M}$ with respect to $\omega / M_{T}$, we obtain

$V_{M}=-2 M_{T} \frac{\omega}{2 f^{2}} C\left(1+\frac{-m^{2}+\omega^{2}}{2 \omega^{2}} \frac{\omega}{M_{T}}\right)+\mathcal{O}\left(\left(\frac{\omega}{M_{T}}\right)^{2}\right)$.

The leading term agrees with the interaction $V_{B}$, except for the trivial normalization factor $2 M_{T}$ :

$$
V_{B}=\frac{V_{M}}{2 M_{T}}
$$

This also indicates, from Eq. (C1), that the scattering amplitudes also coincide up to $\mathcal{O}\left(\left(\omega / M_{T}\right)\right)$ :

$$
t_{B}=\frac{t_{M}}{2 M_{T}}+\mathcal{O}\left(\omega / M_{T}\right)
$$

Thus, we have shown that the formulation given in Sec. II can be applied to the heavy meson target, up to $\mathcal{O}\left(\omega / M_{T}\right)$ corrections. The convergence becomes much better, if we include the next to leading order terms in $V_{B}$ :

$$
V_{B}^{(2)}=-\frac{1}{2 f^{2}} C\left(\sqrt{s}-M_{T}\right) \frac{E_{T}+M_{T}}{2 M_{T}} .
$$

Here $E_{T}$ is the on-shell energy of the baryon, and $\sqrt{s}$ is the total energy in the center-of-mass system. This agrees with the interaction $V_{M}$ up to $\mathcal{O}\left(\left(\omega / M_{T}\right)^{2}\right)$. 
[1] W. M. Yao et al. (Particle Data Group), J. Phys. G 33, 1 (2006).

[2] T. Nakano et al. (LEPS Collaboration), Phys. Rev. Lett. 91, 012002 (2003).

[3] K. H. Hicks, Prog. Part. Nucl. Phys. 55, 647 (2005).

[4] S. K. Choi et al. (Belle Collaboration), Phys. Rev. Lett. 91, 262001 (2003).

[5] B. Aubert et al. (BABAR Collaboration), Phys. Rev. Lett. 90, 242001 (2003).

[6] T. Hyodo, D. Jido, and A. Hosaka, Phys. Rev. Lett. 97, 192002 (2006).

[7] N. Kaiser, P. B. Siegel, and W. Weise, Nucl. Phys. A594, 325 (1995).

[8] E. Oset and A. Ramos, Nucl. Phys. A635, 99 (1998).

[9] B. Krippa, Phys. Rev. C 58, 1333 (1998).

[10] J. A. Oller and U. G. Meissner, Phys. Lett. B 500, 263 (2001).

[11] M. F. M. Lutz and E. E. Kolomeitsev, Nucl. Phys. A700, 193 (2002).

[12] R. H. Dalitz and S. F. Tuan, Ann. Phys. (N.Y.) 10, 307 (1960).

[13] J. H. W. Wyld, Phys. Rev. 155, 1649 (1967).

[14] D. Jido, J. A. Oller, E. Oset, A. Ramos, and U. G. Meissner, Nucl. Phys. A725, 181 (2003).

[15] T. Hyodo, A. Hosaka, E. Oset, A. Ramos, and M. J. Vicente Vacas, Phys. Rev. C 68, 065203 (2003).

[16] V. K. Magas, E. Oset, and A. Ramos, Phys. Rev. Lett. 95, 052301 (2005).

[17] E. E. Kolomeitsev and M. F. M. Lutz, Phys. Lett. B 585, 243 (2004).

[18] S. Sarkar, E. Oset, and M. J. Vicente Vacas, Nucl. Phys. A750, 294 (2005).

[19] M. F. M. Lutz and E. E. Kolomeitsev, Nucl. Phys. A730, 110 (2004).

[20] E. E. Kolomeitsev and M. F. M. Lutz, Phys. Lett. B 582, 39 (2004).

[21] F. K. Guo, P. N. Shen, H. C. Chiang and R. G. Ping, Phys. Lett. B 641, 278 (2006)

[22] F. K. Guo, P. N. Shen and H. C. Chiang, hep-ph/0610008

[23] S. Weinberg, Phys. Rev. Lett. 17, 616 (1966).

[24] Y. Tomozawa, Nuovo Cimento 46A, 707 (1966).

[25] C. Garcia-Recio, M. F. M. Lutz, and J. Nieves, Phys. Lett. B 582, 49 (2004).

[26] V. Bernard, N. Kaiser, and U.-G. Meissner, Int. J. Mod. Phys. E 4, 193 (1995).

[27] G. Ecker, Prog. Part. Nucl. Phys. 35, 1 (1995).

[28] A. Pich, Rep. Prog. Phys. 58, 563 (1995).

[29] T. Hyodo, S. I. Nam, D. Jido, and A. Hosaka, Prog. Theor. Phys. 112, 73 (2004).

[30] J. A. Oller and E. Oset, Phys. Rev. D 60, 074023 (1999).

[31] K. Igi and K.-i. Hikasa, Phys. Rev. D 59, 034005 (1999).

[32] E. Oset, A. Ramos, and C. Bennhold, Phys. Lett. B 527, 99 (2002).
[33] T. Inoue, E. Oset, and M. J. Vicente Vacas, Phys. Rev. C 65, 035204 (2002).

[34] A. Ramos, E. Oset, and C. Bennhold, Phys. Rev. Lett. 89, 252001 (2002).

[35] A. R. Edmonds, Proc. R. Soc. A 268, 567 (1962).

[36] J. J. de Swart, Rev. Mod. Phys. 35, 916 (1963).

[37] S. Sarkar, E. Oset, and M. J. Vicente Vacas, Eur. Phys. J. A24, 287 (2005).

[38] A. Aktas et al. (H1 Collaboration), Phys. Lett. B 588, 17 (2004).

[39] T. D. Cohen, P. M. Hohler, and R. F. Lebed, Phys. Rev. D 72, 074010 (2005).

[40] S. H. Lee, Y. Kwon, and Y. Kwon, Phys. Rev. Lett. 96, 102001 (2006).

[41] V. Kopeliovich, Phys. Lett. B 259, 234 (1991).

[42] D. Diakonov and V. Petrov, Phys. Rev. D 69, 056002 (2004).

[43] V. Kopeliovich, hep-ph/0310071

[44] E. Jenkins and A. V. Manohar, Phys. Rev. Lett. 93, 022001 (2004).

[45] G. 't Hooft, Nucl. Phys. B72, 461 (1974).

[46] E. Witten, Nucl. Phys. B160, 57 (1979).

[47] C. Garcia-Recio, J. Nieves, and L. L. Salcedo, Phys. Rev. D 74, 036004 (2006).

[48] T. D. Cohen and R. F. Lebed, Phys. Rev. D 74, 056006 (2006).

[49] R. F. Dashen, E. Jenkins, and A. V. Manohar, Phys. Rev. D 49, 4713 (1994).

[50] T. D. Cohen and R. F. Lebed, Phys. Rev. D 70, 096015 (2004).

[51] C. G. Callan and I. R. Klebanov, Nucl. Phys. B262, 365 (1985).

[52] N. Itzhaki, I. R. Klebanov, P. Ouyang, and L. Rastelli, Nucl. Phys. B684, 264 (2004).

[53] H. Walliser and H. Weigel, Eur. Phys. J. A 26, 361 (2005).

[54] R. Jackiw, in M.A.B. Bég Memorial Vol., edited by A. Ali and P. Hoodbhoy (World Scientific, Singapore, 1991), pp. 25-42.

[55] N. Isgur and G. Karl, Phys. Rev. D 18, 4187 (1978).

[56] L. Roca, S. Sarkar, V. K. Magas, and E. Oset, Phys. Rev. C 73, 045208 (2006).

[57] T. Hyodo, S. Sarkar, A. Hosaka, and E. Oset, Phys. Rev. C 73, 035209 (2006).

[58] B. Borasoy, R. Nissler, and W. Weise, Phys. Rev. Lett. 94, 213401 (2005).

[59] B. Borasoy, R. Nissler, and W. Weise, Eur. Phys. J. A25, 79 (2005).

[60] J. A. Oller, J. Prades, and M. Verbeni, Phys. Rev. Lett. 95, 172502 (2005).

[61] J. A. Oller, Eur. Phys. J. A 28, 63 (2006).

[62] J. A. Oller, M. Verbeni, and J. Prades, J. High Energy Phys. 09, 079 (2006). 